\title{
Resource Allocation for Wireless-Powered Full-Duplex Relaying Systems with Non-Linear Energy Harvesting Efficiency
}

\author{
Zhongxiang Wei, Member, IEEE, Sumei Sun, Fellow, IEEE, Xu Zhu, Senior Member, IEEE, Dong In \\ Kim, Fellow, IEEE, Derrick Wing Kwan Ng, Senior Member, IEEE
}

\begin{abstract}
In wireless power transfer (WPT)-assisted relaying systems, spectral efficiency (SE) of source-relay link plays a dominant role in system SE performance due to the limited transmission power at the WPT-aided relay. In this paper, we propose a novel protocol for a downlink orthogonal frequency division multiple access (OFDMA) system with a WPT-aided relay operating in full-duplex (FD) decode-and-forward (DF) mode, where the time slot durations of the source-relay and relayusers hops are designed to be dynamic, to enhance the utilization of degrees of freedom and hence the system SE. In particular, a multiple-input and signal-output (MISO) source-relay channel is considered to satisfy the stringent sensitivity of the energy harvesting (EH) circuit at the relay, while a single-input and single-output (SISO) relay-user channel is considered to alleviate the power consumption at the relay node. Taking into account the non-linearity of $\mathrm{EH}$ efficiency, a near-optimal iteration-based dynamic WPT-aided FD relaying (A-FR) algorithm is developed by jointly optimizing the time slot durations, subcarriers, and transmission power at the source and the relay. Furthermore, self-interference generated at the relay is utilized as a vital energy source rather than being canceled, which increases substantially the total energy harvested at the FD relay. We also reveal some implicit characteristics of the considered WPT-aided FD relaying system through intensive discussions. Simulation results confirm that the proposed A-FR achieves a significant enhancement in terms of $\mathrm{SE}$ with different relay's locations and the number of users, compared to the conventional symmetric WPT-aided FD relaying (S-FR) and the time-switching-based WPT-aided FD relaying (TS-FR) benchmarks.
\end{abstract}

Index Terms-Resource allocation, full-duplex relaying, wireless power transfer, non-linear energy harvesting

\section{INTRODUCTION}

Wireless power transfer (WPT)-assisted relaying has attracted much attention due to recent advances in radio frequency-based WPT development [1]-[6]. By harvesting wireless energy through WPT technique, WPT-assisted relays

The paper has been presented in part in the 2017 IEEE 85th Vehicular Technology Conference (VTC Spring), 2017 [3].

Zhongxiang Wei is with the Department of Electrical and Electronics Engineering, University College London, London, U.K. (e-mail: zhongxiang.wei@ucl.ac.uk)

$\mathrm{Xu}$ Zhu is with the University of Liverpool, Liverpool 3GJ, U.K., (e-mail: xuzhu@liverpool.ac.uk).

Sumei Sun is with the Institute for Infocom Research, A*STAR, Singapore. (e-mail: sunsm@i2r.a-star.edu.sg)

Dong In Kim is with the Department of Electrical and Computer Engineering, Sungkyunkwan University, Suwon 16419, Korea. (e-mail: dikim@skku.ac.kr)

D. W. K. Ng is with the School of Electrical Engineering and Telecommunications, The University of New South Wales, NSW 2052, Australia. (e-mail: w.k.ng@unsw.edu.au) can continuously assist communication between sources and users [7] [8] [9]. The early research on WPT-aided relaying systems mainly focused on the three-time-slots half-duplex (HD) transmission [10] [11] [12], cf. Fig. 1(a). In particular, the first time slot is assigned for energy harvesting (EH) at the relay node and another two time slots are required for relaying information signals from the source to the user through the HD relay node. By making use of the power splitting architecture [13] [14], a WPT-assisted HD relaying system requiring only two time slots was considered, cf. Fig. 1(b). In particular, only part of the received signal is decoded in the first time slot, and the remaining signal is harvested and stored by the relay node to supply its own power consumption. Later on, a hybrid timeand power splitting protocol was proposed in [15], cf. Fig. 1(c). Under some strict conditions (Eq. (20), [15]), the hybrid protocol may slightly improve SE performance over the sole time- or power splitting protocol. Hence, the WPT-assisted HD relaying systems either require three time slots (time-splitting based HD structure [10] [11] [12]) or only decode and forward part of the desired signal to the users (power-splitting based HD structure [13] [14]), leading to a low spectral efficiency (SE) performance caused by the low resource utilization in HD relaying protocols.

Various techniques have been proposed to improve the SE performance. Among them, full-duplex (FD) relaying protocols can approximately double the SE over HD relaying [16] [17] [18]. Also, [19] extended FD relaying into a multiuser scenario by orthogonal frequency division multiple access (OFDMA) technique, which has been widely used in 3GPP Long Term Evolution or IEEE 802.11 LAN systems [20]. Recently, there is a growing interest in integrating WPT-aided relaying and FD techniques to enable simultaneous wireless information and power transfer with high SE [21]. In the literature, a two-hop WPT-aided FD relay system usually requires two time slots [23] [24], where the relay works in FD mode in one time slot but HD mode in the other time slot, cf. Fig. 1(e). This is because if a relay works in FD mode at all time, self-interference cancellation and power splitting for $\mathrm{EH}$ must be performed simultaneously, which imposes extremely high complexity on transceiver design [22]. Also, the energy of self-interference cannot be harvested in this case. Hence, a two-slot WPT-aided relaying structure is more practical and strikes a good trade-off between system performance and circuit complexity. In [23] and [24], SE maximization in FD WPT-aided relaying systems was investigated, where the EH 
and the information transmission are decoupled into two time slots, i.e., one for $\mathrm{EH}$ at the relay node and the other for FD transmission. Joint precoding design at the source and relay was demonstrated in [25] for FD amplify-and-forward (AF) relaying systems, where the multiple users are powered by WPT from a relay node. With the FD relaying protocol for information transfer in [23]-[25], only two time slots are needed and the SE is improved over the HD counterparts.

In the aforementioned WPT-assisted FD relaying systems, however, the generated self-interference is considered as an undesired element and hence stringent self-interference cancellation is needed. In contrast, self-interference in WPT-assisted FD relaying systems [26] [27] can be utilized for EH. For example, the source transmits signals to the relay in the first time slot, while the relay forwards the re-encoded signal to the users and concurrently harvests wireless energy originated from both the source and the relay's self-interference in the second time slot, cf. Fig. 1(d). However, uniform time slot durations were assumed for the two hops in [26] and [27], and system end-to-end SE may be limited by the relay-users bottleneck. It is because the available power at the relay is generally much lower than that at the source, due to the wireless channel attenuation and the low EH efficiency. Hence, the received signal-to-interference-plus-noise ratio (SINR) of the relay-users hop is much lower than that of the sourcerelay hop, and a large portion of system resource allocated to improve SE for the source-relay hop is partially wasted by the symmetric transmission mechanism. Besides, only a signal-user and single-carrier scenario with an over-simplified linear EH efficiency model was considered in [23]-[27], which cannot capture the turn-on effect at low input power case as well as the saturation effect at high input power case.

Motivated by the aforementioned challenges, we propose a novel protocol for OFDMA systems with a WPT-aided relay working in FD decode-and-forward (DF) mode. Our work is different in the following aspects:

a) We design dynamic time slot durations for the multipleinput and single-output (MISO) source-relay link and the single-input and single-output (SISO) relay-user link, which enhances the utilizations of the degrees of freedom and improves the end-to-end SE over the works in [23] and [27]. Besides, by allowing dynamic durations of the two time slots, much more energy can be harvested at the relay than the works in [23] and [27].

b) We take into account the non-linear characteristic of EH circuits, since the over-simplified linear conversion model is highly inaccurate with respect to practical EH circuits. Besides, we investigate a multiuser system, which is more general in communication networks, such as in Internet-ofThings (IoT) and wireless sensor networks, while only singleuser scenarios were considered in the previous work [23]-[27].

c) Based on the developed dynamic relaying protocol, we propose a near-optimal iteration-based resource allocation algorithm to maximize the overall system SE by performing joint allocation of time slot durations, subcarriers for multiple users, and transmission power at the source and relay. The closed-forms of optimal resource allocation policy are given. We find that the proposed joint optimization can achieve a

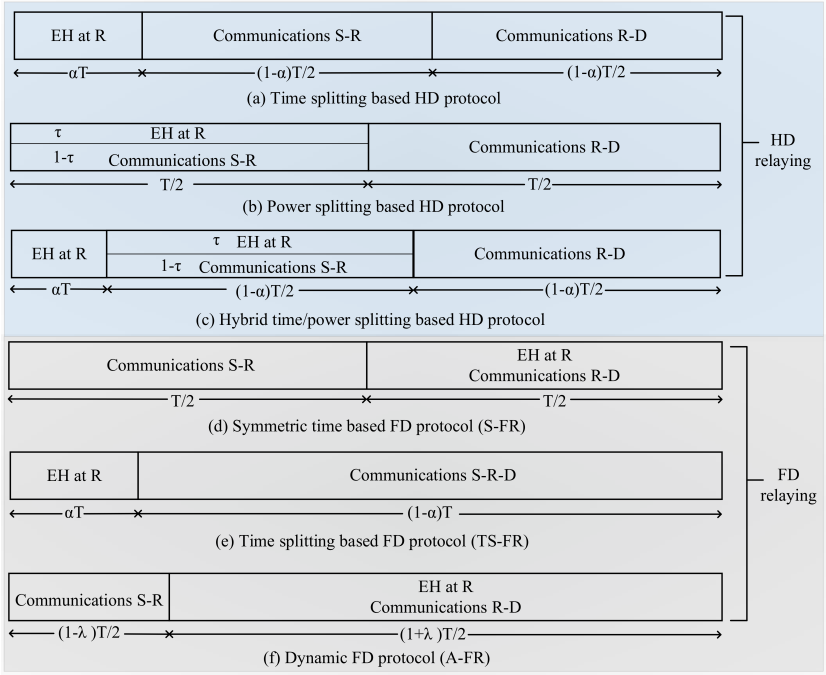

Fig. 1. A schematic diagram of different WPT-aided relaying protocols, where $\alpha$ and $\tau$ denote the time- and power splitting factors, respectively. (a)-(c) Time splitting, power splitting, and hybrid time/power splitting based HD protocols. (d) Symmetric time based FD protocol (S-FR). (e) Time splitting based FD protocol (TS-FR). (d) The proposed dynamic FD protocol (A-FR).

significant SE enhancement compared to the symmetric WPTaided FD relaying (S-FR) [27] and the time-switching WPTaided FD relaying (TS-FR) schemes [23].

d) An intensive discussion of WPT-aided FD relaying systems is demonstrated, i.e., the relative time slot durations of the two hops, impact of self-interference on the power allocation policy, diversity and complexity, revealing some implicit characteristics of WPT-aided FD relay systems.

Notations: Matrices and vectors are represented by boldface capital and lower case letters, respectively. $|\cdot|$ denotes the absolute value of a complex scalar. $\|\cdot\|_{2}$ denotes the Euclidean vector norm. $(\cdot)^{H}$ and $\operatorname{Tr}(\cdot)$ denote the Hermitian transpose and trace of matrix or vector. Superscripts $k$ or $n$ denote the users' or subcarrier indexes, respectively. Operator $[\cdot]^{+}$denotes $[x]^{+}=\max \{0, x\}$ while operator $[\cdot]_{[-1,1]}$ denotes $[x]_{[-1,1]}=$ $x$ if $-1 \leq x \leq 1$, and $[x]_{[-1,1]}=-1$ if $x<-1$ or $[x]_{[-1,1]}=1$ or $1<x$. Operator $\nabla$ denotes derivative calculator.

\section{System Model And Problem Formulation}

\section{A. System Model}

We consider a downlink OFDMA system with $K$ users and $N$ subcarriers, and the communication between the source and the multiple users is assisted by a relay in between. We assume the multiple users cannot communicate with the source directly caused by the blockage and the channel attenuation [23] [27], cf. Fig. 1(f) and Fig. 2. This is also a typical communication scenario in wireless networks, such as in cellular networks [19], wireless sensor networks [28] and wireless local networks [29]. For example, in wireless sensor networks, the source transmits information to a cluster head (relay) and the cluster head forwards the information to its cluster members (multiple users) through a broadcast channel [28]. The relay is charged from wireless energy through WPT technique [10]. To address the EH sensitivity issue at the relay 

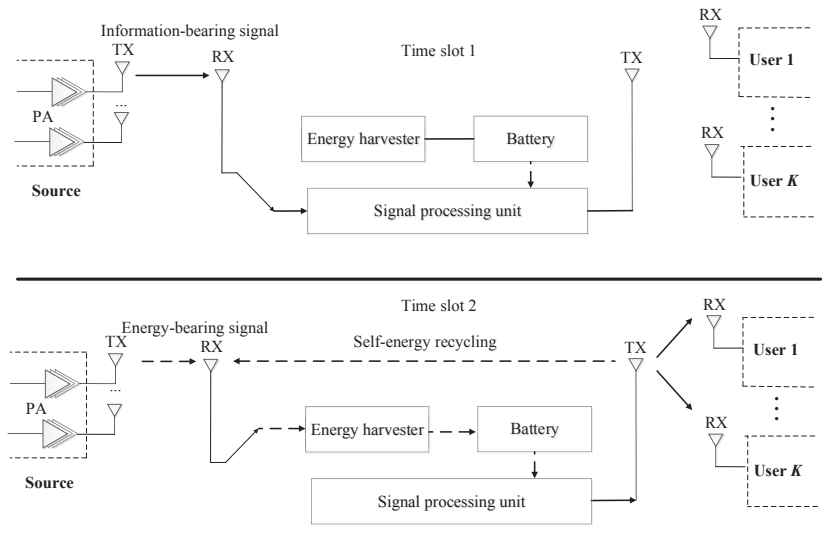

Fig. 2. Illustration of a WPT-aided FD DF relaying multiuser system with dynamic and asymmetric time durations, where dashed and solid lines indicate energy-bearing and information-bearing signals, respectively. The first and second sub-figures show the transmission protocol in $T_{1}$ and $T_{2}$, respectively.

and make WPT reliable over a long communication distance, the source is equipped with $N_{T}$ multiple transmission antennas [30]. Besides, due to the size and battery limitations, the relay is equipped with two antennas, one for receiving information and $\mathrm{EH}$, and the other for transmitting signal to the multiple users, while the users are equipped with one reception antenna. Channel state information (CSI) can be obtained by channel estimation in the training phase. Assume that the multiple users utilize orthogonal pilot sequences, which allows the pilots of different users can be distinguished. First, the relay and multiple users transmit their own pilot signal, respectively. Hence, the CSI of source-relay link can be obtained at the source, and CSI of relay-users links can be obtained at the relay. Especially, the pilot signal of the relay is also echoed back to the relay itself, and self-interference channel is also obtained at the relay node. Then, the relay can forward CSI indicator to the source to indicate the CSI of relay-users and self-interference links. Hence, CSI of all links in a two-hop FD relaying system is acquired at the source [19] [31].

\section{B. Energy Harvesting Model}

Field measurement indicates that practical $\mathrm{EH}$ circuit is quite non-linear [32]. Non-linear EH efficiency has significant impact on the overall harvested power at the relay, which further affects the system SE. Hence, we adopt a more practical logistic function based EH model [33]. The introduced nonlinear EH model captures the turn-on effect at lower input power case and saturation effect at high input power case. If the input power is low, the relay is unable to harvest power. On the other hand, if saturation power at the relay is achieved, the overall harvested power cannot be increased with more input power. Define $p_{i n}$ as the overall input power at the relay's receiver, the harvested power $P_{h}$ can be given by

$$
P_{h}\left(p_{i n}\right)=\frac{\Psi-P_{s a t} \Omega}{1-\Omega},
$$

where $\Psi=\frac{P_{s a t}}{1+e^{-a\left(p_{i n}-b\right)}}$ is the logistic function with respect to the input variable $p_{i n} . P_{\text {sat }}$ denotes the maximum saturated
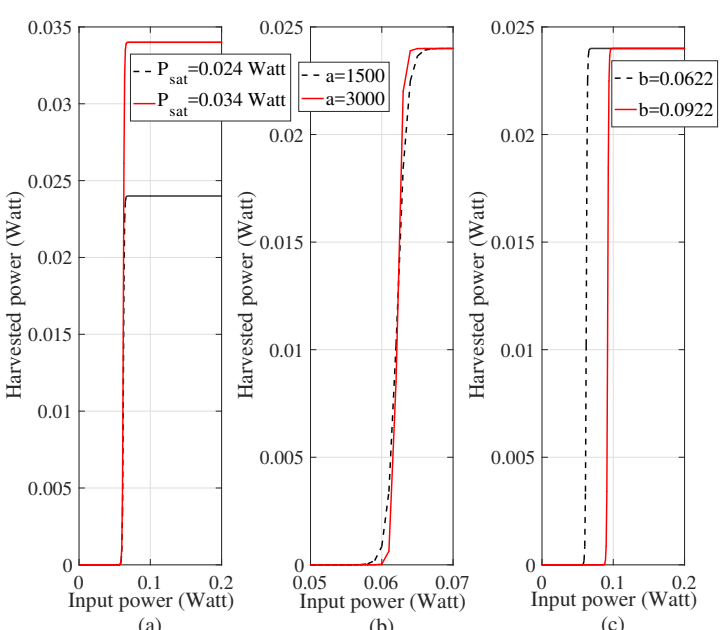

(c)

Fig. 3. Impact of the parameters $P_{s a t}, a$ and $b$ on the EH performance. (a) $a=1500, b=0.0622$. (b) $P_{\text {sat }}=0.024$ Watt, $b=0.0622$. (C) $P_{\text {sat }}=0.024$ Watt, $a=1500$.

EH power due to an excessively large input power. Parameter $\Omega$ is calculated as $\Omega=\frac{1}{1+e^{a b}}$, which ensures a zero-input/zerooutput response for EH circuit. The parameters $a$ and $b$ are related to the detailed circuit specifications such as the resistance, capacitance, and diode turn-on voltage. The values of the parameters can be determined by curve-fitting.

Fig. 3 demonstrates the impact of the different values of the parameters $P_{\text {sat }}, a$ and $b$ on the EH circuit performance. It can be seen that the value of $P_{\text {sat }}$ directly determines the saturation power, and a higher value of $P_{\text {sat }}$ denotes a higher saturation power. The parameter $a$ determines the slope of the EH curve. In particular, a higher value of $a$ means that an EH receiver needs a higher value of input power to activate its EH circuit but achieves its saturation power quickly. The parameter $b$ determines sensitivity of the EH receiver, and a higher value of $b$ means that an EH receiver is less sensitive to input power and needs a higher input power to activate its EH circuit. In summary, the parameters $P_{s a t}, a$ and $b$ jointly define the characteristics of an EH circuit.

\section{Channel Model}

Now, we introduce the adopted notations in the paper. Define power allocation matrices at the source $\boldsymbol{p}_{\boldsymbol{s}}=\left[p_{s, k, n}\right]_{K \times N}$ and at the relay $\boldsymbol{p}_{\boldsymbol{r}}=\left[p_{r, k, n}\right]_{K \times N}$, whose elements $p_{s, k, n}$ and $p_{r, k, n}$ indicate the transmission power assigned at the source and the relay for the $k$-th user on subcarrier $n$, respectively. Define subcarrier allocation matrix $\boldsymbol{\beta}=\left[\beta_{k, n}\right]_{K \times N}$, where $\beta_{k, n}=1$ indicates subcarrier $n$ is assigned to the $k$-th user otherwise $\beta_{k, n}=0$.

In the first time slot, the received signal on subcarrier $n$ for user $k$ at the relay can be given as

$$
r_{k, n, 1}[i]=\sqrt{p_{s, k, n} l_{s r}} \boldsymbol{h}_{s r, n} \boldsymbol{w}_{n} x_{k, n}[i]+n_{r, n}[i],
$$

where $i$ denotes the $i$-th symbol index. $x_{k, n}[i]$ is the information-bearing signal for the $k$-th user on subcarrier $n$ 
with unit power $\mathbb{E}\left\{\left|x_{k, n}[i]\right|^{2}\right\}=1$. $\boldsymbol{h}_{s r, n} \in \mathbb{C}^{1 \times N_{T}}$ indicates the channel gain of the source-relay link on subcarrier $n$, and $l_{s r}$ captures the large-scale fading of the first hop. $\boldsymbol{w}_{n} \in \mathbb{C}^{N_{T} \times 1}$ denotes the precoding vector for the subcarrier $n$ at the source. $n_{r, n}[i]$ is the noise introduced on subcarrier $n$ at the relay.

Evidently, the power allocation parameter for user $k$ on subcarrier $n$ has been captured by the variable $p_{s, k, n}, \forall k \in$ $K, n \in K$, and the source-relay is a MISO channel. Hence, the optimal precoding design of $\boldsymbol{w}_{n}$ can be given as $\boldsymbol{w}_{n}=\frac{\boldsymbol{h}_{s r, n}^{H}}{\left\|\boldsymbol{h}_{s r, n}\right\|_{2}}$, where the superscript $(\cdot)^{H}$ denotes the Hermitian and the operator $\|\cdot\|_{2}$ denotes 2-norm of a vector, respectively. Now, the received signal on subcarrier $n$ for user $k$ at the relay can be re-written as

$$
\begin{aligned}
r_{k, n, 1}[i] & =\sqrt{p_{s, k, n} l_{s r}} \frac{\boldsymbol{h}_{s r, n} \boldsymbol{h}_{s r, n}^{H}}{\left\|\boldsymbol{h}_{s r, n}\right\|_{2}} x_{k, n}[i]+n_{r, n}[i] \\
& =\sqrt{p_{s, k, n} l_{s r}}\left\|\boldsymbol{h}_{s r, n}\right\|_{2} x_{k, n}[i]+n_{r, n}[i],
\end{aligned}
$$

As can be seen, transmission diversity at the source is significantly improved by introducing multiple antennas at the source. As a result, receiving power at the relay is increased and hence the stringent sensitivity of $\mathrm{EH}$ receiver is addressed. Now, the overall $\mathrm{SE}$ of the first hop in the first time slot $T_{1}$ is given as

$$
C_{S R}=\frac{T_{1}}{T} \sum_{k=1}^{K} \sum_{n=1}^{N} \beta_{k, n} \log _{2}\left(1+p_{s, k, n} \gamma_{s r, k, n}\right),
$$

where $\beta_{k, n}$ denotes that subcarrier $n$ is allocated for user $k$ or not. $\gamma_{s r, k, n}=\frac{\left\|\boldsymbol{h}_{s r, n}\right\|_{2}^{2} l_{s r}}{N_{0, r} B}$ denotes the equivalent channel-tonoise ratio $(\mathrm{CNR})$ of the source-relay link on subcarrier $n$ for the $k$-th user. $N_{0, r}$ is the noise power spectral density at the relay node and $B$ is the bandwidth of each subcarrier.

In the second time slot with duration $T_{2}$, the re-encoded signal at the relay is forwarded to the multiple users. For simplicity, subcarrier mapping is not considered in this paper. The received signal by the $k$-th user on subcarrier $n$ is given by

$$
y_{k, n}[i]=\sqrt{p_{r, k, n} l_{r d, k}} h_{r d, k, n} x_{k, n}[i]+z_{k, n}[i],
$$

where $h_{r d, k, n}$ denotes the channel gain of the relay-user $k$ on subcarrier $n$, and $l_{r d, k}$ captures the effects of large-scale fading of the relay-user $k . z_{k, n}[i]$ is the noise introduced at user $k$. Therefore, the system SE in $T_{2}$ is calculated as

$$
C_{R D}=\frac{T_{2}}{T} \sum_{k=1}^{K} \sum_{n=1}^{N} \beta_{k, n} \log _{2}\left(1+p_{r, k, n} \gamma_{r d, k, n}\right),
$$

where $\gamma_{r d, k, n}=\frac{\left|h_{r d, n}\right|^{2} l_{r d, k}}{N_{0, k} B}$ denotes the CNR of the relay-user $k$ on subcarrier $n$ and $N_{0, k}$ denotes the noise power spectral density at user $k$. Finally, the overall end-to-end SE can be expressed as

$$
C=\min \left\{C_{S R}, C_{R D}\right\}
$$

Concurrently with the information-bearing signal $x_{k, n}[i]$ being forwarded by the relay, energy-bearing signal $x_{k, n}^{(e)}[i]$ is sent from the source for EH operation [27] in the second time slot. Hence, the composite received signal at the relay for user $k$ on subcarrier $n$ in $T_{2}$ is calculated as

$$
\begin{aligned}
r_{k, n, 2}[i]= & \sqrt{p_{s, k, n} l_{s r}}\left\|\boldsymbol{h}_{s r, n}\right\|_{2} x_{k, n}^{(e)}[i]+ \\
& \sqrt{p_{r, k, n} l_{r r}} h_{r r, n} x_{k, n}[i-\tau]+n_{r, n}[i],
\end{aligned}
$$

where $\mathbb{E}\left\{\left|x_{k, n}^{(e)}[i]\right|^{2}\right\}=1$ without loss of generality. $h_{r r, n}$ is the small-scale fading on subcarrier $n$ and $l_{r r}$ is large-scale fading of self-interference channel. $\tau$ is the processing symbol delay at relay node ${ }^{1}$.

Since the channel condition of $h_{r r, n}$ and the transmitted signal $x_{k, n}[i-\tau]$ are known by the system, we can adjust the phase of the energy-bearing signal $x_{k, n}^{(e)}[i]$, and make the received signals $x_{k, n}^{(e)}[i]$ and $x_{k, n}[i-\tau]$ aligned at the relay. Hence, we have $x_{k, n}^{(e)}[i]=x_{k, n}[i-\tau] e^{j<h_{r r, n}},\left(\angle h_{r r, n}\right.$ denotes the phase of the complex number $h_{r r, n}$, respectively) and the harvested energy is maximized at the relay [27], as briefly discussed in Proposition 1.

Proposition 1: Since OFDMA is adopted as the multiuser access technique and the received signal at the relay comes from the source and relay itself, the overall collected power at the relay is summed over all the subcarriers and users. Hence, the maximum input power at the relay is calculated as

$$
p_{i n}=\sum_{k=1}^{K} \sum_{n=1}^{N} \beta_{k, n}\left(p_{s, k, n}\left\|\boldsymbol{h}_{s r, n}\right\|_{2}^{2} l_{s r}+p_{r, k, n}\left|h_{r r, n}\right|^{2} l_{r r}\right) \text {. }
$$

Again, since the second time slot $T_{2}$ is utilized for $\mathrm{EH}$ at the relay node, the maximal energy harvested at the relay is calculated as $E_{h}=T_{2} P_{h}\left(p_{i n}\right)$.

\section{Problem Formulation}

Since the overall SE in Eq. (7) is optimized by jointly allocating variables $T_{1}, T_{2}, \boldsymbol{p}_{\boldsymbol{s}}, \boldsymbol{p}_{\boldsymbol{r}}$, and $\boldsymbol{\beta}$, we now define $C\left(T_{1}, T_{2}, \boldsymbol{p}_{\boldsymbol{s}}, \boldsymbol{p}_{\boldsymbol{r}}, \boldsymbol{\beta}\right)$ as the overall $\mathrm{SE}$ (in $\mathrm{bps} / \mathrm{Hz}$ ) in the objective function of $\mathrm{P} 1$. For the power consumption at the relay, it is mainly consumed by its power amplifier and signal processing circuit. In particular, the power consumed by power amplifier is given by $\frac{\sum_{k=1}^{K} \sum_{n=1}^{N} \beta_{k, n} p_{r, k, n}}{\eta_{r}}$, where $\eta_{r}$ denotes power amplifier efficiency at the relay. On the other hand, as indicated in [27] [36], for the devices with small size and low signal processing requirement (such as relay, wireless sensors and tags), the power consumed by circuit is much lower than that consumed by power amplifier. Hence, the circuit power at the relay $p_{\text {cir }}$ is assumed to be a constant. Besides, considering the low conversion efficiency at the relay and long communication distance between the source and relay, the harvested energy at the relay is much lower than its battery

\footnotetext{
${ }^{1}$ There are some methods to improve the EH efficiency by hardware design at relay node, such as improving mutual coupling between relay's two antennas [26] or directing the self-interference power from relay's transmit antenna into the relay's battery [34]. More information can be found in [26], [34].
} 
storage limit. Moreover, the harvested energy at the relay is immediately used for forwarding information to multiple users, and battery storage limit at the relay is hardly to be violated. Hence, the battery storage constraint is omitted from the problem formulation [31]. Accordingly, the optimization problem is formulated as

$$
\begin{array}{ll}
P 1: & \max _{T_{1}, T_{2}, \boldsymbol{p}_{s}, \boldsymbol{p}_{\boldsymbol{r}}, \boldsymbol{\beta}} C\left(T_{1}, T_{2}, \boldsymbol{p}_{\boldsymbol{s}}, \boldsymbol{p}_{\boldsymbol{r}}, \boldsymbol{\beta}\right) \\
\text { s.t. } & (C 1): 0 \leq \beta_{k, n} p_{s, k, n}, \forall n \in N, k \in K, \\
& (C 2): \frac{\sum_{k=1}^{K} \sum_{n=1}^{N} \beta_{k, n} p_{s, k, n}}{\eta_{s}} \leq p_{\max }, \\
& (C 3): 0 \leq \beta_{k, n} p_{r, k, n}, \forall n \in N, k \in K, \\
& (C 4): \frac{\sum_{k=1}^{K} \sum_{n=1}^{N} \beta_{k, n} p_{r, k, n}}{\eta_{r}}+p_{c i r} \leq P_{h}\left(p_{i n}\right), \\
& (C 5): T_{1}+T_{2}=T, \\
& (C 6): \sum_{k=1}^{K} \beta_{k, n}=1, \\
& (C 7): \beta_{k, n} \in\{0,1\}, \forall n \in N, k \in K,
\end{array}
$$

where constraints $(C 1)-(C 2)$ indicate the allocated transmission power at the source should be non-negative and upper bounded by the overall available power supply $p_{\max }$, with power amplifier efficiency $\eta_{s}$ at the source. Constraints $(C 3)$ $(C 4)$ indicate the consumed power at the relay should be nonnegative and upper bounded by the overall harvested power $P_{h}\left(p_{i n}\right)$. (C5) means the sum of $T_{1}$ and $T_{2}$ equals to the total time duration $T$. $(C 6)$ and $(C 7)$ guarantee that each subcarrier is not shared by multiple users. The allocation of time slots $T_{1}$ and $T_{2}$, subcarrier matrix $\boldsymbol{\beta}$, transmission power matrices $\boldsymbol{p}_{\boldsymbol{s}}$ and $\boldsymbol{p}_{\boldsymbol{r}}$ should be jointly allocated, subjected to constraints $(C 1)-(C 7)$.

\section{Resource Allocation Design}

The objective function of $\mathrm{P} 1$ is not convex and its constraints do not confine a convex set. Furthermore, binary variable $\boldsymbol{\beta}$ is involved in P1 and imposes further difficulty in optimization design. Hence, to solve the non-convex problem $P 1$ in a low computational complexity, the original problem is first transformed into $P 2$. Then, by exploiting the properties of the $P 2$, the problem is further simplified into $P 3$, as detailed in Subsection III-A. Optimal results and discussion are presented in Subsection III-B and Subsection III-C.

\section{A. Transformation of the Optimization Problem}

We first handle the min function in the objective function, according to the following Proposition 2.

Proposition 2: Since the end-to-end SE performance of two-hop DF relaying system is limited by its bottleneck link, $P 1$ is optimized only when $\frac{T_{1}}{T} \sum_{k=1}^{K} \sum_{n=1}^{N} \beta_{k, n} \log _{2}(1+$ $\left.p_{s, k, n} \gamma_{s r, k, n}\right)=\frac{T_{2}}{T} \sum_{k=1}^{K} \sum_{n=1}^{N} \beta_{k, n} \log _{2}\left(1+p_{r, k, n} \gamma_{r d, k, n}\right)$, which can be easily proved by a counter example. For example, if the maximum end-to-end $\mathrm{SE}$ is achieved when the SE of the first link is higher than that of the second link, one can always reduce the value of
$T_{1}$ to compensate $T_{2}$ and further improve the end-toend SE performance, until $\frac{T_{1}}{T} \sum_{k=1}^{K} \sum_{n=1}^{N} \beta_{k, n} \log _{2}(1+$ $\left.p_{s, k, n} \gamma_{s r, k, n}\right)=\frac{T_{2}}{T} \sum_{k=1}^{K} \sum_{n=1}^{N} \beta_{k, n} \log _{2}\left(1+p_{r, k, n} \gamma_{r d, k, n}\right)$ is obtained. Therefore, the original optimization problem $\min \left\{\frac{T_{1}}{T} \sum_{k=1}^{K} \sum_{n=1}^{N} \beta_{k, n} \log _{2}(1+\right.$ $\left.\left.p_{s, k, n} \gamma_{s r, n}\right), \frac{T_{2}}{T} \sum_{k=1}^{K} \sum_{n=1}^{N} \beta_{k, n} \log _{2}\left(1+p_{r, k, n} \gamma_{r d, k, n}\right)\right\}$ is equivalent to maximizing $\frac{T_{1}}{T} \sum_{k=1}^{K} \sum_{n=1}^{N} \beta_{k, n} \log _{2}(1+$ $\left.p_{s, k, n} \gamma_{s r, k, n}\right)+\frac{T_{2}}{T} \sum_{k=1}^{K} \sum_{n=1}^{N} \beta_{k, n} \log _{2}\left(1+p_{r, k, n} \gamma_{r d, k, n}\right)$, with an additional constraint $\frac{T_{1}}{T} \sum_{k=1}^{K} \sum_{n=1}^{N} \beta_{k, n} \log _{2}(1+$ $\left.p_{s, k, n} \gamma_{s r, k, n}\right)=\frac{T_{2}}{T} \sum_{k=1}^{K} \sum_{n=1}^{N} \beta_{k, n} \log _{2}\left(1+p_{r, k, n} \gamma_{r d, k, n}\right)$.

Then the transformed problem is given as

$$
\begin{aligned}
& P 2: \max _{T_{1}, T_{2}, \boldsymbol{p}_{\boldsymbol{s}}, \boldsymbol{p}_{\boldsymbol{r}}, \boldsymbol{\beta}} \frac{T_{1}}{T} \sum_{k=1}^{K} \sum_{n=1}^{N} \beta_{k, n} \log _{2}\left(1+p_{s, k, n} \gamma_{s r, k, n}\right)+ \\
& \frac{T_{2}}{T} \sum_{k=1}^{K} \sum_{n=1}^{N} \beta_{k, n} \log _{2}\left(1+p_{r, k, n} \gamma_{r d, k, n}\right) \\
& \text { s.t. }(C 1)-(C 7), \\
& \begin{aligned}
(C 8): & \frac{T_{1}}{T} \sum_{k=1}^{K} \sum_{n=1}^{N} \beta_{k, n} \log _{2}\left(1+p_{s, k, n} \gamma_{s r, k, n}\right)= \\
& \frac{T_{2}}{T} \sum_{k=1}^{K} \sum_{n=1}^{N} \beta_{k, n} \log _{2}\left(1+p_{r, k, n} \gamma_{r d, k, n}\right) .
\end{aligned}
\end{aligned}
$$

Please note an additional constraint (C8) is imposed for validating the transformation from $\mathrm{P} 1$ to $\mathrm{P} 2$ [35].

We then relax the binary constraint $(C 7)$. In particular, the element $\beta_{k, n}$ is relaxed to a continuous real number within the interval $[0,1]$, which can be interpreted as the time sharing factor of subcarrier $n$ among the multiple users [38]. Such a relaxation is near-optimal when the number of subcarriers [38] is large ${ }^{2}$. As a result, constraint $(C 1)$ can be transformed into $(\tilde{C} 1): 0 \leq p_{s, k, n}, \forall n \in N, k \in K$; Constraint $(C 2)$ can be transformed into $(\tilde{C} 2): \sum_{k=1}^{K} \sum_{n=1}^{N} p_{s, k, n} \leq p_{\max }$; Constraint (C3) can be transformed into $(\tilde{C} 3): 0 \leq p_{r, k, n}, \forall n \in N, k \in$ $K$; Constraint $(C 7)$ can be transformed into $(\tilde{C} 7): \beta_{k, n}=$ $[0,1], \forall n \in N, k \in K$, and constraints $(C 8)$ can be transformed into $(\tilde{C} 8): \frac{T_{1}}{T} \sum_{k=1}^{K} \sum_{n=1}^{N} \beta_{k, n} \log _{2}\left(1+\frac{p_{s, k, n} \gamma_{s r, k, n}}{\beta_{k, n}}\right)=$ $\frac{T_{2}}{T} \sum_{k=1}^{K} \sum_{n=1}^{N} \beta_{k, n} \log _{2}\left(1+\frac{p_{r, k, n} \gamma_{r d, k, n}}{\beta_{k, n}}\right)$. Now the difficulty lies in $(C 4)$. Since the input power at the relay originates from both the source and relay itself, for simplicity, we define $P_{h}\left(\boldsymbol{p}_{\boldsymbol{s}}, \boldsymbol{p}_{\boldsymbol{r}}\right)$ as the harvested power with resource allocation policy $\boldsymbol{p}_{\boldsymbol{s}}$ and $\boldsymbol{p}_{\boldsymbol{r}}$. Substituting Eq. (9) into (C4) yields

$$
(\tilde{C} 4): \frac{\sum_{k=1}^{K} \sum_{n=1}^{N} p_{r, k, n}}{\eta_{r}}+p_{c i r} \leq P_{h}\left(\boldsymbol{p}_{\boldsymbol{s}}, \boldsymbol{p}_{\boldsymbol{r}}\right),
$$

where $P_{h}\left(\boldsymbol{p}_{\boldsymbol{s}}, \boldsymbol{p}_{\boldsymbol{r}}\right)$ is specifically given as $P_{h}\left(\boldsymbol{p}_{\boldsymbol{s}}, \boldsymbol{p}_{\boldsymbol{r}}\right)=$ $\frac{\frac{P_{s a t}}{1+\exp \left(-a\left(\sum_{k=1}^{K} \Sigma_{n=1}^{N}\left(p_{s, k, n}\left|h_{s r, n}\right|^{2} l_{s r}+p_{r, k, n}\left|h_{r r, n}\right|^{2} l_{r r}\right)-b\right)\right.}-P_{s a t} \Omega}{1-\Omega}$ according to Proposition 1. Evidently, the right-hand side of Eq. (12) is a differentiable concave function. Therefore, successive convex approximation method can be adopted

\footnotetext{
${ }^{2}$ Actually, time-sharing based subcarrier relaxation is practical and has been extensively adopted for OFDMA multiuser systems, regardless of the convexity of the objective function. More information is found in [37], [39].
} 
to handle the problem, and the following inequality always holds for any feasible point:

$$
\begin{aligned}
& P_{h}\left(\boldsymbol{p}_{\boldsymbol{s}}, \boldsymbol{p}_{\boldsymbol{r}}\right) \leq P_{h}\left(\boldsymbol{p}_{\boldsymbol{s}}^{(j)}, \boldsymbol{p}_{\boldsymbol{r}}^{(j)}\right) \\
& +\sum_{k=1}^{K} \sum_{n=1}^{N} \nabla_{p_{s, k, n}} P_{h}\left(\boldsymbol{p}_{\boldsymbol{s}}^{(j)}, \boldsymbol{p}_{\boldsymbol{r}}^{(j)}\right)\left(p_{s, k, n}-p_{s, k, n}^{(j)}\right) \\
& +\sum_{k=1}^{K} \sum_{n=1}^{N} \nabla_{p_{r, k, n}} P_{h}\left(\boldsymbol{p}_{\boldsymbol{s}}^{(j)}, \boldsymbol{p}_{\boldsymbol{r}}^{(j)}\right)\left(p_{r, k, n}-p_{r, k, n}^{(j)}\right),
\end{aligned}
$$

where the operator $\nabla$ denotes the derivation calculation and $j$ denotes the iteration index. It follows that, for a given value of $\left(\boldsymbol{p}_{\boldsymbol{s}}^{(j)}, \boldsymbol{p}_{\boldsymbol{r}}^{(j)}\right)$ at the $j$-th iteration, solving the optimization problem after replacing the right-hand side of constraint $(\tilde{C} 4)$ by (13) leads to an upper bound of the optimal results. In order to tighten this upper bound, we develop an iterative algorithm, where an intermediate solution is obtained with the replaced constraint $\frac{\sum_{k=1}^{K} \sum_{n=1}^{N} p_{r, k, n}}{\eta_{r}}+p_{c i r} \leq$ $P_{h}\left(\boldsymbol{p}_{\boldsymbol{s}}^{(j)}, \boldsymbol{p}_{\boldsymbol{r}}^{(j)}\right)+\sum_{k=1}^{K} \sum_{n=1}^{N} \nabla p_{s, k, n} P_{h}\left(\boldsymbol{p}_{\boldsymbol{s}}^{(j)}, \boldsymbol{p}_{\boldsymbol{r}}^{\eta_{r}}\right)\left(p_{s, k, n}-\right.$ $\left.p_{s, k, n}^{(j)}\right)+\sum_{k=1}^{K} \sum_{n=1}^{N} \nabla p_{r, k, n} P_{h}\left(\boldsymbol{p}_{\boldsymbol{s}}^{(j)}, \boldsymbol{p}_{\boldsymbol{r}}^{(j)}\right)\left(p_{r, k, n}-p_{r, k, n}^{(j)}\right)$. These steps are repeated iteratively until convergence. Since the right-hand side of constraint $(\tilde{C} 4)$ is concave, the iterative algorithm always converges to the optimum results with respect to the original problem formulation with polynomial computational complexity. Now all the constraints have been handled. We propose Proposition 3 to show the concavity of the objective function.

Proposition 3: The equivalent end-to-end SE $\frac{T_{1}}{T} \sum_{k=1}^{K} \sum_{n=1}^{N} \beta_{k, n} \log _{2}\left(1+\frac{p_{s, k, n} \gamma_{s r, k, n}}{\beta_{k, n}}\right)+$

$\frac{T_{2}}{T} \sum_{k=1}^{K} \sum_{n=1}^{N} \beta_{k, n} \log _{2}\left(1+\frac{p_{r, k, n} \gamma_{r d, k, n}}{\beta_{k, n}}\right)$ is a jointly concave function with respect to all the variables $T_{1}, T_{2}, p_{s, k, n}, p_{r, k, n}$, $\beta_{k, n}, \forall n \in N, k \in K$.

The proof of Proposition 3 is presented in Appendix A.

\section{B. Solution of the Optimization Problem}

After some mathematical manipulations, the problem at hand can be equivalently written as

$$
\begin{aligned}
& P 3: \max _{T_{1}, T_{2}, \boldsymbol{p}_{\boldsymbol{s}}, \boldsymbol{p}_{\boldsymbol{r}}, \boldsymbol{\beta}} \frac{T_{1}}{T} \sum_{k=1}^{K} \sum_{n=1}^{N} \beta_{k, n} \log _{2}\left(1+\frac{p_{s, k, n} \gamma_{s r, k, n}}{\beta_{k, n}}\right)+ \\
& \frac{T_{2}}{T} \sum_{k=1}^{K} \sum_{n=1}^{N} \beta_{k, n} \log _{2}\left(1+\frac{p_{r, k, n} \gamma_{r d, k, n}}{\beta_{k, n}}\right) \\
& \text { s.t. }(\tilde{C} 1)-(\tilde{C} 3),(C 5),(\tilde{C} 6)-(\tilde{C} 8) \text {, } \\
& (\tilde{C} 4): \frac{\sum_{k=1}^{K} \sum_{n=1}^{N} p_{r, k, n}}{\eta_{r}}+p_{\text {cir }} \leq P_{\text {harvest }}\left(\boldsymbol{p}_{\boldsymbol{s}}^{(j)}, \boldsymbol{p}_{\boldsymbol{r}}^{(j)}\right)+ \\
& \sum_{k=1}^{K} \sum_{n=1}^{N} \nabla p_{s, k, n} P_{\text {harvest }}\left(\boldsymbol{p}_{\boldsymbol{s}}^{(j)}, \boldsymbol{p}_{\boldsymbol{r}}^{(j)}\right)\left(p_{s, k, n}-p_{s, k, n}^{(j)}\right)+ \\
& \sum_{k=1}^{K} \sum_{n=1}^{N} \nabla_{p_{r, k, n}} P_{\text {harvest }}\left(\boldsymbol{p}_{\boldsymbol{s}}^{(j)}, \boldsymbol{p}_{\boldsymbol{r}}^{(j)}\right)\left(p_{r, k, n}-p_{r, k, n}^{(j)}\right) \text {. }
\end{aligned}
$$

Because P3' dual problem now is a concave problem and is satisfied with the Slater's constraint qualification, the KarushKuhn-Tucker (KKT) conditions can be adopted. To this end, the Lagrangian function of P3 is written by (15), where $\lambda, \mu, \phi, \rho$, and $\psi_{n}(\forall n \in N)$ are the Lagrange multipliers corresponding to the multiple constraints, and $\mu \geq 0, \phi \geq$ $0, \rho \geq 0,1 \geq \lambda \geq-1$, and $\psi_{n} \geq 0(\forall n \in N)$ can be confirmed by the KKT conditions. Let $\mu^{*}, \phi^{*}, \rho^{*}, \lambda^{*}$ and $\psi_{n}^{*}$ (for $\forall n \in N$ ) denote the optimal Lagrange multipliers, we obtain Eqs. (16) and (17), respectively. Since P3 is maximized when $\frac{T_{1}}{T} \sum_{k=1}^{K} \sum_{n=1}^{N} \beta_{k, n} \log _{2}\left(1+\frac{p_{s, k, n} \gamma_{s r, k, n}}{\beta_{k, n}}\right)=$ $\frac{T_{2}}{T} \sum_{k=1}^{K} \sum_{n=1}^{N} \beta_{k, n} \log _{2}\left(1+\frac{p_{r, k, n} \gamma_{r d, k, n}}{\beta_{k, n}}\right)$, the following equality is always satisfied

$$
\frac{T_{1}^{*}}{1-\lambda^{*}}=\frac{T_{2}^{*}}{1+\lambda^{*}} .
$$

Substituting (C5) into (18) yields

$$
T_{1}^{*}=\frac{1-\lambda^{*}}{2} T \text {. }
$$

and the optimal duration of $T_{2}^{*}$ can be expressed as

$$
T_{2}^{*}=\frac{1+\lambda^{*}}{2} T
$$

Similarly, taking the derivative of $L$ with respect to $p_{s, k, n}$ and $p_{r, k, n}$ yields Eqs. (21) and (22), respectively. Substituting (19) and (20) into (21) and (22), the optimal power allocation at the source can be given as

$$
\begin{aligned}
& p_{s, k, n}^{*}= \\
& {\left[\frac{\left(1-\lambda^{*}\right)^{2} \beta_{k, n}^{*}}{2 \ln (2)\left(\frac{\mu^{*}}{\eta_{s}}-\phi^{*} \nabla p_{s, k, n} P_{h}\left(\boldsymbol{p}_{\boldsymbol{s}}^{(j)}, \boldsymbol{p}_{\boldsymbol{r}}^{(j)}\right)\right)}-\frac{\beta_{k, n}^{*}}{\gamma_{s r, k, n}}\right]^{+},}
\end{aligned}
$$

where the operator $[x]^{+}=\max \{0, x\}$. Also, we have the optimal power allocation at the relay as

$$
\begin{aligned}
& p_{r, k, n}^{*}= \\
& {\left[\frac{\left(1+\lambda^{*}\right)^{2} \beta_{k, n}^{*}}{2 \ln (2) \phi^{*}\left(\frac{1}{\eta_{r}}-\nabla p_{r, k, n} P_{h}\left(\boldsymbol{p}_{\boldsymbol{s}}^{(j)}, \boldsymbol{p}_{\boldsymbol{r}}^{(j)}\right)\right)}-\frac{\beta_{k, n}^{*}}{\gamma_{r d, k, n}}\right]^{+} .}
\end{aligned}
$$

Taking the derivative of $L$ with respect to $\beta_{k, n}$ yields

$$
\begin{aligned}
& \frac{\partial L}{\partial \beta_{k, n}}= \frac{\left(1-\lambda^{*}\right) T_{1}}{T} \log _{2}\left(1+\frac{p_{s, k, n}^{*} \gamma_{s r, k, n}}{\beta_{k, n}^{*}}\right)- \\
& \frac{\left(1-\lambda^{*}\right) T_{1}^{*} p_{s, k, n}^{*} \gamma_{s r, k, n}}{\ln (2)\left(\beta_{k, n}^{*}+p_{s, k, n}^{*} \gamma_{s r, k, n}\right) T}+ \\
& \frac{\left(1+\lambda^{*} T_{2}^{*}\right.}{T} \log _{2}\left(1+\frac{p_{r, k, n}^{*} \gamma_{r d, k, n}}{\beta_{k, n}^{*}}\right)- \\
& \frac{\left(1+\lambda^{*}\right) T_{2}^{*} p_{r, k, n}^{*} \gamma_{r d, k, n}}{\ln (2)\left(\beta_{k, n}^{*}+p_{r, k, n}^{*} \gamma_{r d, k, n}\right) T}-\psi_{n}^{*}=\left\{\begin{array}{l}
<0, \beta_{k, n}^{*}=0 \\
\geq 0, \beta_{k, n}^{*}=1
\end{array} .\right.
\end{aligned}
$$

Since $\beta_{k, n}$ is either 0 or 1 , by substituting (19), (20), (23) and (24) into (25), the subcarrier $n$ will be assigned to the user $k$ satisfying

$$
\beta_{k, n}^{*}=\left\{\begin{array}{l}
1, k=k^{*} \\
0, k \neq k^{*},
\end{array}\right.
$$




$$
\begin{aligned}
L= & \frac{T_{1}}{T} \sum_{k=1}^{K} \sum_{n=1}^{N} \beta_{k, n} \log _{2}\left(1+\frac{p_{s, k, n} \gamma_{s r, k, n}}{\beta_{k, n}}\right)+\frac{T_{2}}{T} \sum_{k=1}^{K} \sum_{n=1}^{N} \beta_{k, n} \log _{2}\left(1+\frac{p_{r, k, n} \gamma_{r d, k, n}}{\beta_{k, n}}\right)-\mu\left(\sum_{k=1}^{K} \sum_{n=1}^{N} \frac{p_{s, k, n}}{\eta_{s}}-p_{\text {max }}\right) \\
& \lambda\left(\frac{T_{1}}{T} \sum_{k=1}^{K} \sum_{n=1}^{N} \beta_{k, n} \log _{2}\left(1+\frac{p_{s, k, n} \gamma_{s r, k, n}}{\beta_{k, n}}\right)-\frac{T_{2}}{T} \sum_{k=1}^{K} \sum_{n=1}^{N} \beta_{k, n} \log _{2}\left(1+\frac{p_{r, k, n} \gamma_{r d, k, n}}{\beta_{k, n}}\right)\right) \\
& \phi\left(\frac{\sum_{k=1}^{K} \sum_{n=1}^{N} p_{r, k, n}}{\eta_{r}}+p_{\text {cir }}-P_{\text {harvest }}\left(\boldsymbol{p}_{\boldsymbol{s}}^{(i)}, \boldsymbol{p}_{\boldsymbol{r}}^{(i)}\right)-\sum_{k=1}^{K} \sum_{n=1}^{N} \nabla_{p_{s, k, n}} P_{\text {harvest }}\left(\boldsymbol{p}_{\boldsymbol{s}}^{(i)}, \boldsymbol{p}_{\boldsymbol{r}}^{(i)}\right)\left(p_{s, k, n}-p_{s, k, n}^{(i)}\right)\right. \\
& \left.-\sum_{k=1}^{K} \sum_{n=1}^{N} \nabla_{p_{r, k, n}} P_{\text {harvest }}\left(\boldsymbol{p}_{\boldsymbol{s}}^{(i)}, \boldsymbol{p}_{\boldsymbol{r}}^{(i)}\right)\left(p_{r, k, n}-p_{r, k, n}^{(i)}\right)\right)-\rho\left(T_{1}+T_{2}-T\right)-\sum_{n=1}^{N} \psi_{n}\left(\sum_{k=1}^{K} \beta_{k, n}-1\right),
\end{aligned}
$$

$$
\frac{\partial L}{\partial T_{1}}=\frac{1-\lambda^{*}}{T} \sum_{k=1}^{K} \sum_{n=1}^{N} \beta_{k, n}^{*} \log _{2}\left(\frac{1+p_{s, k, n}^{*} \gamma_{s r, k, n}}{\beta_{k, n}^{*}}\right)-\rho^{*}\left\{\begin{array}{l}
<0, T_{1}^{*}=0 \\
=0, T_{1}^{*}>0
\end{array}\right.
$$

$$
\frac{\partial L}{\partial T_{2}}=\frac{1+\lambda^{*}}{T} \sum_{k=1}^{K} \sum_{n=1}^{N} \beta_{k, n}^{*} \log _{2}\left(1+\frac{p_{r, k, n}^{*} \gamma_{r d, k, n}}{\beta_{k, n}^{*}}\right)-\rho^{*}\left\{\begin{array}{l}
<0, T_{2}^{*}=0 \\
=0, T_{2}^{*}>0
\end{array}\right.
$$

$$
\frac{\partial L}{\partial p_{s, k, n}}=\frac{\left(1-\lambda^{*}\right) T_{1}^{*} \gamma_{s r, k, n} \beta_{k, n}^{*}}{\ln (2)\left(\beta_{k, n}^{*}+p_{s, k, n}^{*} \gamma_{s r, k, n}\right) T}-\frac{\mu^{*}}{\eta_{s}}+\phi^{*} \nabla_{p_{s, k, n}} P_{h}\left(\boldsymbol{p}_{\boldsymbol{s}}^{(j)}, \boldsymbol{p}_{\boldsymbol{r}}^{(j)}\right)\left\{\begin{array}{l}
<0, p_{s, k, n}^{*}=0 \\
=0, p_{s, k, n}^{*}>0
\end{array}\right.
$$

where $k^{*}=\underset{g}{\operatorname{argmax}}$

$$
\begin{aligned}
& \frac{\left(1-\lambda^{*}\right)^{2}}{2}\left[\log _{2}\left(\frac{\left(1-\lambda^{*}\right)^{2}}{2 \ln 2} \frac{\gamma_{s r, k, n}}{\left(\frac{\mu^{*}}{\eta_{s}}-\phi^{*} \nabla_{p_{s, k}, n} P_{h}\left(\boldsymbol{p}_{s}^{(j)}, \boldsymbol{p}_{r}^{(j)}\right)\right.}\right)\right]^{+} \\
& {\left[\frac{(1-\lambda)^{2} \gamma_{s r, k, n}-2 \ln 2\left(\frac{\mu^{*}}{\eta_{s}}-\phi^{*} \nabla p_{s, k, n} P_{h}\left(\boldsymbol{p}_{s}^{(j)}, \boldsymbol{p}_{r}^{(j)}\right)\right)}{2 \gamma_{s r, k, n}}\right]^{+}} \\
& \frac{\left(1+\lambda^{*}\right)^{2}}{2}\left[\operatorname { l o g } _ { 2 } \left(\frac{\left(1+\lambda^{*}\right)^{2}}{2 \ln 2} \frac{\gamma_{r d, k, n}}{\left.\phi^{*}\left(\frac{1}{\eta_{r}}-\nabla_{p_{r, k, n} P_{h}\left(\boldsymbol{p}_{s}^{(j)}, \boldsymbol{p}_{r}^{(j)}\right)}\right)\right]^{+}}\right.\right. \\
& {\left[\frac{(1+\lambda)^{2} \gamma_{r d, k, n}-2 \ln 2 \phi^{*}\left(\frac{1}{\eta_{r}}-\nabla_{p_{r, k, n}} P_{h}\left(\boldsymbol{p}_{s}^{(j)}, \boldsymbol{p}_{r}^{(j)}\right)\right)}{2 \gamma_{r d, k, n}}\right]^{+} .}
\end{aligned}
$$

Finally,

the optimal values of $p_{s, k, n}$ and $p_{r, k, n}$ are given by

$$
\begin{aligned}
& p_{s, k, n}^{*}= \\
& {\left[\frac{\left(1-\lambda^{*}\right)^{2}}{2 \ln (2)\left(\frac{\mu^{*}}{\eta_{s}}-\phi^{*} \nabla p_{s, k, n} P_{h}\left(\boldsymbol{p}_{\boldsymbol{s}}^{(j)}, \boldsymbol{p}_{\boldsymbol{r}}^{(j)}\right)\right)}-\frac{1}{\gamma_{s r, k, n}}\right]^{+},} \\
& p_{r, k, n}^{*}= \\
& {\left[\frac{\left(1+\lambda^{*}\right)^{2}}{2 \ln (2) \phi^{*}\left(\frac{1}{\eta_{r}}-\nabla p_{r, k, n} P_{h}\left(\boldsymbol{p}_{\boldsymbol{s}}^{(j)}, \boldsymbol{p}_{\boldsymbol{r}}^{(j)}\right)\right)}-\frac{1}{\gamma_{r d, k, n}}\right]^{+} .}
\end{aligned}
$$

Now we adopt the gradient method to update the multipliers $\mu, \phi$ and $\lambda$, while $\psi_{n}(\forall n \in N)$ and $\rho$ have been omitted naturally in deriving subcarrier allocation and time duration, as shown in Eqs. (19), (20) and (26). The rules of updating multipliers are calculated by (29), shown on the top of next page, where $\epsilon_{1}(i), \epsilon_{2}(i)$ and $\epsilon_{3}(i)$ are the corresponding step sizes [38] at the $i$-th inner iteration. In order to guarantee the - convergence of the algorithm, the values of the step sizes $\epsilon_{1}(i)$, $\epsilon_{2}(i)$ and $\epsilon_{3}(i)$ should satisfy $\sum_{i=1}^{\infty} \epsilon(i)=\infty$ and $\lim _{i \rightarrow \infty} \epsilon(i)=0$.

$+\quad$ Now we are able to devise the whole algorithm. The proposed - algorithm has two layers. In the inner layer, the algorithm calculates the intermediate optimal resource allocation results with the given values of $\boldsymbol{p}_{\boldsymbol{s}}^{(j)}$ and $\boldsymbol{p}_{\boldsymbol{r}}^{(j)}$. After each iteration, the values of $\boldsymbol{p}_{\boldsymbol{s}}^{(j)}$ and $\boldsymbol{p}_{\boldsymbol{r}}^{(j)}$ are updated in the outer layer. Two steps iteratively run until convergence. The whole algorithm is summarized in Algorithm 1.

\section{Properties Discussion}

In this subsection, some important properties in dynamic WPT-aided FD relaying systems are discovered.

Remark 1: In wireless-powered relaying systems, equallength time slot allocation is strictly suboptimal. In particular, for a WPT-aided relay, its available transmission power is generally much lower than that at the source due to signal attenuation from source to relay and low EH efficiency. As a result, the SINR of the relay-users link is practically lower than that of the source-relay link, which creates a performance bottleneck to the end-to-end SE. Hence, allocating equal-length time slot cannot alleviate the situation, and the

$$
\frac{\partial L}{\partial p_{r, k, n}}=\frac{\left(1+\lambda^{*}\right) T_{2}^{*} \gamma_{r d, k, n} \beta_{k, n}^{*}}{\ln (2)\left(\beta_{k, n}^{*}+p_{r, k, n}^{*} \gamma_{r d, k, n}\right) T}-\phi^{*}\left(\frac{1}{\eta_{r}}-\nabla p_{r, k, n} P_{h}\left(\boldsymbol{p}_{\boldsymbol{s}}^{(j)}, \boldsymbol{p}_{\boldsymbol{r}}^{(j)}\right)=\left\{\begin{array}{l}
<0, p_{r, k, n}^{*}=0 \\
=0, p_{r, k . n}^{*}>0
\end{array}\right.\right.
$$




$$
\begin{aligned}
& \lambda(i+1)=\left[\lambda(i)+\epsilon_{1}(i) \times\left(\sum_{k=1}^{K} \sum_{n=1}^{N} \beta_{k, n} \log _{2}\left(1+\frac{p_{s, k, n} \gamma_{s r, k, n}}{\beta_{k, n}}\right)-\sum_{k=1}^{K} \sum_{n=1}^{N} \beta_{k, n} \log _{2}\left(1+\frac{p_{r, k, n} \gamma_{r d, k, n}}{\beta_{k, n}}\right)\right)\right]_{[-1,1]} ; \\
& \mu(i+1)=\left[\mu(i)+\epsilon_{2}(i) \times\left(\sum_{k=1}^{K} \sum_{n=1}^{N} p_{s, k, n}-p_{m a x}\right)\right]^{+} ; \phi(i+1)=\left[\phi(i)+\epsilon_{3}(i) \times\left(\frac{\sum_{k=1}^{K} \sum_{n=1}^{N} p_{r, k, n}}{\eta_{r}}+p_{c i r}-\right.\right. \\
& \left.\left.P_{h}\left(\boldsymbol{p}_{\boldsymbol{s}}^{(j)}, \boldsymbol{p}_{\boldsymbol{r}}^{(j)}\right)-\sum_{k=1}^{K} \sum_{n=1}^{N} \nabla_{s_{s, k, n}} P_{h}\left(\boldsymbol{p}_{\boldsymbol{s}}^{(j)}, \boldsymbol{p}_{\boldsymbol{r}}^{(j)}\right)\left(p_{s, k, n}-p_{s, k, n}^{(j)}\right)-\sum_{k=1}^{K} \sum_{n=1}^{N} \nabla_{p_{r, k, n}} P_{h}\left(\boldsymbol{p}_{\boldsymbol{s}}^{(j)}, \boldsymbol{p}_{r}^{(j)}\right)\left(p_{r, k, n}-p_{r, k, n}^{(j)}\right)\right)\right]^{+} ;
\end{aligned}
$$

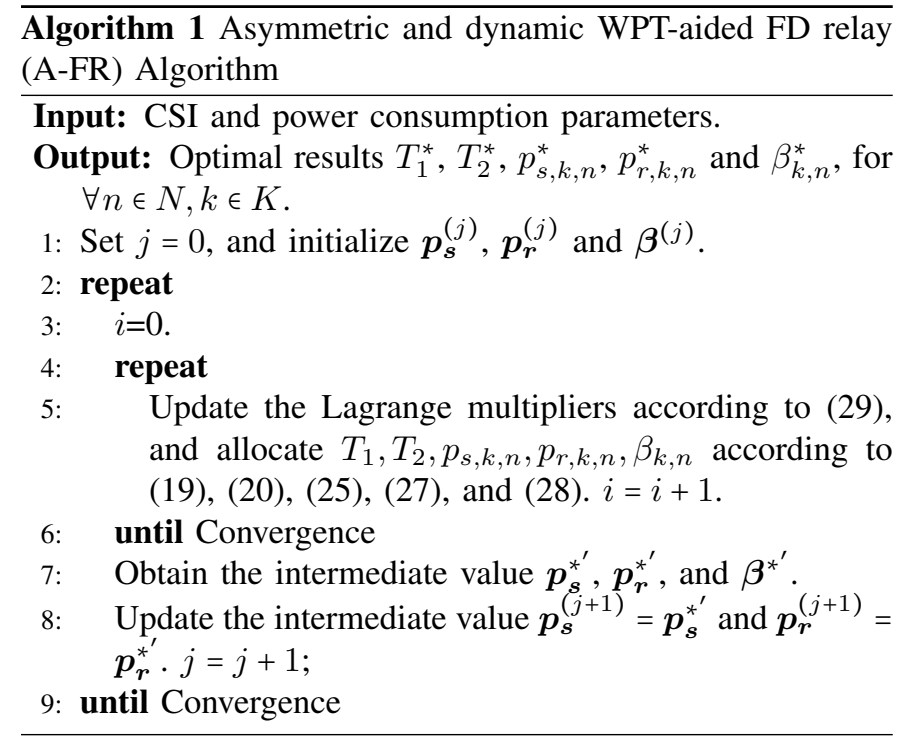

bottleneck relay-users link significantly limits the end-to-end SE performance. The proposed algorithm adjusts the lengths of the two time slots adaptively, and $\lambda \geq 0$ can be determined by the algorithm, as can be seen from (29). In summary, according to the relative SINRs of the two links, the proposed system can adaptively adjust the values of $T_{1}$ and $T_{2}$ to maximize the overall end-to-end SE.

Remark 2: In dynamic WPT-aided FD relaying systems, the optimal transmission power at the source follows the waterfilling approach. As can be seen from (27), the water level is given by $\frac{\left(1-\lambda^{*}\right)^{2}}{2 \ln (2)\left(\frac{\mu^{*}}{n_{s}}-\phi^{*} \nabla p_{s, k, n} P_{h}\left(\boldsymbol{p}_{s}^{(j)}, \boldsymbol{p}_{r}^{(j)}\right)\right)}$. Evidently, the system prefers to assign more power on the subcarrier with higher value of $\gamma_{s r, k, n}$.

Remark 3: For the proposed A-FR system, transmission power allocation at the relay is completely different from that in conventional grid-powered FD relaying systems. As demonstrated by Eq. (28), the power allocation policy at the relay node is jointly determined by values of the channel frequency responses $\left|h_{r r, k, n}\right|$ and $\left|h_{r d, k, n}\right|$. Now we investigate how $\left|h_{r r, k, n}\right|$ (inside the $P_{h}(\cdot)$ function) affects the power allocation. With the same channel response of $\left|h_{r d, k, n}\right|$, $\forall n \in N, k \in K$, the A-FR prefers to assign more power on the subcarrier with a higher value of $\left|h_{r r, k, n}\right|$. In contrast, more transmission power is allocated to the subcarrier with a lower value of $\left|h_{r r, k, n}\right|$ in conventional FD grid-powered relay systems [41]. It is because for the proposed algorithm, self-interference is exploited for $\mathrm{EH}$ and more energy can be harvested with a higher value of $\left|h_{r r, k, n}\right|$, while self- interference is treated as an undesired element in [41] and [42], and therefore the gain of the self-interference channel should be as low as possible.

Remark 4: In traditional FD relaying systems, selfinterference is considered as an undesired element and has to be suppressed [41]. Therefore, passive suppression is always performed in the first stage to suppress self-interference. To this end, the transmitter and receiver at the relay should be separated from each other, which requires stringent antenna deployment. However, in our system, a more severe selfinterference can improve the amount of harvested energy. Therefore, the requirement on antenna deployment could be eased. Besides, since self-interference cancellation operation can be omitted at the relay node, hardware circuit design can be efficiently simplified as well.

Remark 5: When source-users direct link is presented, in the first time slot the received signal at the user is given as

$$
y_{k, n, 1}=\sqrt{p_{s, k, n} l_{s k}} \boldsymbol{h}_{s d, k, n} \boldsymbol{w}_{n} x_{k, n}[i]+z_{k, n}[i],
$$

where $l_{s k}$ captures the large-scale fading from the source to user $k . \boldsymbol{h}_{s d, k, n}$ indicates the channel gain of the source-user $k$ link on subcarrier $n . \boldsymbol{w}_{n}=\frac{\boldsymbol{h}_{s r, n}^{H}}{\left\|\boldsymbol{h}_{s r, n}^{H}\right\|_{2}}$ is the precoding vector at the source as indicated by Eq. (3). In the second time slot, the relay forwards the re-encoded signal to multiple users while concurrently receiving energy-bearing signal from the source. Hence, the received signal at the user $k$ is given as

$$
\begin{aligned}
y_{k, n, 2}= & \sqrt{p_{r, k, n} l_{r d, k}} h_{r d, k, n} x_{k, n}[i]+ \\
& \sqrt{p_{s, k, n} l_{s k}} \boldsymbol{h}_{s d, k, n} \boldsymbol{w}_{n} x_{k, n}^{(e)}[i]+z_{k, n}[i],
\end{aligned}
$$

where the second term denotes the energy-bearing signal from the source and is treated as noise at the user end. Finally at the $k$-th user end, it can apply maximum ratio combining (MRC) to align the two signals $y_{k, n, 1}$ and $y_{k, n, 2}$ such that

$$
y_{k, n}=\boldsymbol{q}_{k, n, 1} y_{k, n, 1}+\boldsymbol{f}_{k, n, 2} y_{k, n, 2},
$$

where $\boldsymbol{q}_{k, n, 1}=\left(\boldsymbol{h}_{s d, k, n} \boldsymbol{w}_{n}\right)^{H}$ and $\boldsymbol{f}_{k, n, 2}=h_{r d, k, n}^{H}$ are the combining weights in the two time slots. Hence, assuming $T_{1}=T_{2}$, the received signal at the $k$-th user on subcarrier $n$ is finally given by 
$y_{k, n}=$

$\left(\sqrt{p_{s, k, n} l_{s k}}\left\|\boldsymbol{h}_{s d, k, n} \boldsymbol{w}_{k, n}\right\|_{2}+\sqrt{p_{r, k, n} l_{k d, k}}\left|h_{r d, k, n}\right|\right) x_{k, n}[i]+$ $\sqrt{p_{s, k, n} l_{s k}} h_{r d, k, n}^{H} \boldsymbol{h}_{s d, k, n} \boldsymbol{w}_{k, n} x_{k, n}^{(e)}[i]+\left(\boldsymbol{q}_{k, n, 1}+\boldsymbol{f}_{k, n, 2}\right) z_{k, n}[i]$,

where the second term represents the interference caused by the energy-bearing signal from the source, and the third term represents the equivalent noise. From (33), it can be seen although the $k$-th user can apply MRC to improve receiving diversity, the receiving quality may be interfered by the energy-bearing signal from the source. Optimal resource allocation with direct link will be our future research topic.

Remark 6: The overall computational complexity can be given as $\mathbb{O}\left(N K l_{\text {inner }} l_{\text {outer }}\right)$, where $l_{\text {inner }}$ represents the number of iterations in the inner layer, i.e., updating multipliers according to (29). On the other hand, $l_{\text {outer }}$ represents the number of iterations in the outer layer, i.e., updating intermediate variables $\boldsymbol{p}_{s}^{(j)}$ and $\boldsymbol{p}_{r}^{(j)}$ after the $j$-th iteration. The iteration behaviour is further demonstrated by Fig. 12 .

Since the proposed algorithm iteratively runs in a two-layer structure, hereby we analyze the convergence in both inner and outer layers, respectively. In the inner layer, P3 is solved by its dual decomposition and its convergence is guaranteed (i.e., updating multipliers in Eq. (29)), thanks to the concave dual problem of the problem P3. On the other hand, intermediate variables $\boldsymbol{p}_{s}^{(j)}$ and $\boldsymbol{p}_{r}^{(j)}$ are updated at the outer layer, which is originated from utilizing successive convex approximation to handle the non-convex constraint (C4). The original from of $P_{h}\left(\boldsymbol{p}_{s}, \boldsymbol{p}_{r}\right)$ in (C4) is jointly concave with respect to its variables, which is further upper bounded by its first-order Taylor approximation (the right hand of (13)). By iteratively updating the value of $\boldsymbol{p}_{s}^{(j)}$ and $\boldsymbol{p}_{r}^{(j)}$ in each iteration, the linear approximation gradually approaches the original form (C4) and convergence can be guaranteed [33].

Remark 7: Without loss of generality, assume the $k$-th user is allocated with $N_{k}$ subcarriers, where $N_{k} \leq N$. Hence, multiple copies $\left(x_{k, 1}[i], x_{k, 2}[i], \ldots, x_{k, N_{k}}[i]\right)$ are received and combined by the $k$-th user through frequency diversity. By the proposed system, multiple users are accessed by OFDMA technique and there is no multiuser interference. Hence, its diversity gain is approximately calculated as [43]

$$
\begin{aligned}
& d \approx-\lim _{T_{s n r} \rightarrow \infty} \frac{\log \left(C_{2 N_{k}-1}^{N_{k}} \frac{1}{2 N_{k}}\left(\frac{1}{T_{s n r}}\right)^{N_{k}}\right)}{\log \left(T_{s n r}\right)} \\
& =\lim _{T_{s n r} \rightarrow \infty} N_{k}-\frac{\log \left(C_{2 N_{k}-1}^{N_{k}} \frac{1}{2 N_{k}}\right)}{\log \left(T_{s n r}\right)}=N_{k} \leq N,
\end{aligned}
$$

where $C_{2 N_{k}-1}^{N_{k}}=\frac{\left(2 N_{k}-1\right) !}{\left(N_{k}-1\right) ! N_{k} !}$ and $T_{s n r}$ denotes transmit SNR. Eq. (34) indicates that the $k$-th user's diversity is related to the number of the allocated subcarriers and upper bounded by the total number of subcarriers $N$.

\section{Numerical Results}

We use numerical results to verify our analysis in Section IV.The simulation setup is shown in TABLE I. The path loss
(PL) model at $900 \mathrm{MHz}$ scenario [26] [36] is adopted, which is a common PL model employed for WPT-aided systems. Without loss of generality, we set $d_{S D}=d_{S R}+d_{R D}=100 \mathrm{~m}$, where $d_{S D}, d_{S R}$, and $d_{R D}$ are the distances of the sourceusers, the source-relay and the relay-users links. Assume that the source-relay and relay's echo links follow Rician distribution with $5 \mathrm{~dB}$ Rician factor, which enables a strong LOS component for improving received power at the relay. For the relay-users links, since the users are more likely in a scatter-rich environment, the relay-users links are assumed to follow Rayleigh channel, which is a common assumption in two-hop relaying systems [41]. In particular, in Fig. 7, we demonstrate SE performance under imperfect CSI acquisition of the relay-users link. It is because the source and relay are generally fixed, and the CSI of the source-relay and relay's self-echo channels is much more accurate than that of the relay-users link. Denote $h_{r d, k, n}=h_{r d, k, n}+e_{r d, k, n}$, where $h_{r d, k, n}$ is the estimated channel of the $k$-th user on subcarrier $n$ while $e_{r d, k, n}$ denotes channel estimation error following standard Gaussian distribution, such that $e_{r d, k, n} \sim \mathbb{C N}\left(0, \sigma_{e}^{2}\right)$, $\forall k \in K, n \in N$.

Besides, we select the two following algorithms as benchmarks: 1) S-FR system [27], where $T_{1}=T_{2}=\frac{T}{2}$. 2) TS-FR system [23], where the relay works in EH mode in $T_{1}$, and then the overall harvested energy is used for relaying signals from the source to the multiple users in $T_{2}$. Since self-interference is considered as harmful element, it is suppressed at the relay node and the amount canceled in [23] is set to $80 \mathrm{~dB}$. The values of the time slot durations, $T_{1}$ and $T_{2}$, are calculated according to ([23], Eq. (16)).

\section{A. SE Performance}

Fig. 4 shows the SE performance under different transmission powers. The proposed A-FR achieves a substantially higher SE performance, around 1.1-1.8 times higher than the S-FR and 2.5 times higher than the TS-FR systems. This is because the SE of the two links can be well balanced in the AFR system, benefiting from a higher utilization of degrees of freedom. In contrast, the S-FR system only allocates uniform time slots for its source and relay, whereas the effective SINR of each relay-user hop is significantly lower than that of the first hop. As a result, the system SE is significantly limited by the bottleneck relay-users link, and the resources allocated for

TABLE I

SiMULATION SETUP

\begin{tabular}{c|c}
\hline Number of subcarrier $N$ and users $K$ & 128,10 \\
\hline Bandwidth $B$ & $156 \mathrm{kHz}$ \\
\hline Noise power spectral density & $-174 \mathrm{dBm} / \mathrm{Hz}$ \\
\hline Central carrier frequency & $900 \mathrm{MHz}$ \\
\hline Path loss (PL) model & $\mathrm{PL}=31.7+10 \xi \log _{10}\left(d / d_{0}\right)$ \\
\hline PL exponent $\xi$ & $\xi=2.5$ \\
\hline Reference distance $d_{0}$ & $d_{0}=1 \mathrm{~m}$ \\
\hline Saturation power $P_{s a t}$ & 0.024 Watt \\
\hline Non-linear EH model parameters & $a=1500$, and $b=0.0013$ \\
\hline Communication distance & $d_{S D}=d_{S R}+d_{R D}=100 \mathrm{~m}$ \\
\hline Fading of source-relay and echo link & Rician with $5 \mathrm{~dB}$ Rician factor \\
\hline Fading of relay-users link & Rayleigh distribution \\
\hline Drain efficiency $\eta_{s}$ and $\eta_{r}$ & $60 \%$ \\
\hline
\end{tabular}




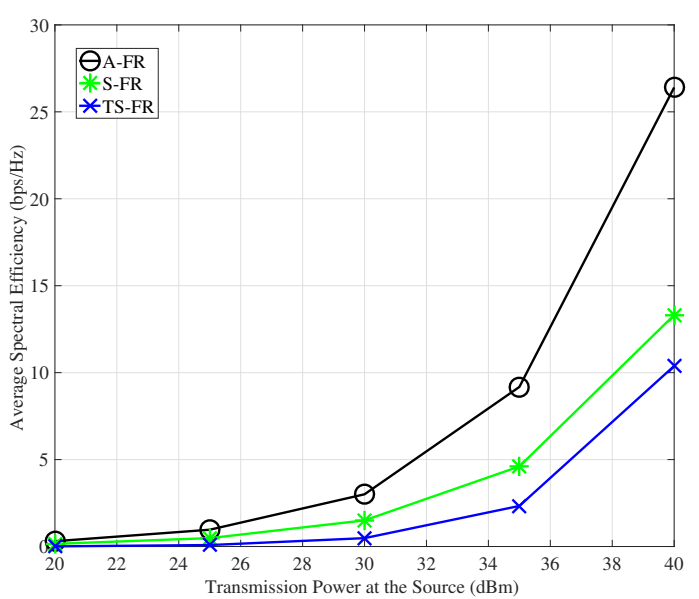

Fig. 4. Impact of the transmission power at the source on SE performance with $d_{S R}=d_{R D}=50 \mathrm{~m}$.

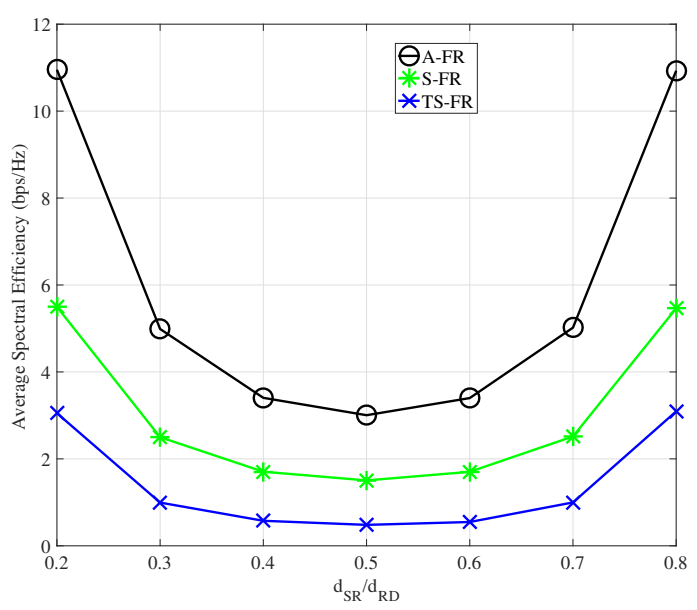

Fig. 5. Impact of the normalized source-relay distance on SE performance with $d_{S D}=100 \mathrm{~m}$ and $p_{s}=30 \mathrm{dBm}$.

improving SE is wasted partially. Besides, the TS-FR shows the worst performance in terms of SE. In fact, its relay node solely harvests energy from the source while self-interference is not exploited for $\mathrm{EH}$, leading to a low transmission power available at the relay node. Hence, the SE of the TS-FR is inevitably bottlenecked by the insufficient power supply.

Fig. 5 shows how the SE performance is affected by the relay's positions. The highest $\mathrm{SE}$ is demonstrated by the proposed A-FR system, which outperforms S-FR and TS-FR systems by 2 times and 4 times, respectively. It is because with different relay's positions, the SINRs of source-relay and relay-user vary, while the A-FR system can adaptively allocate the lengths of two time slots to equalize the SE of the two hops to maximize the system performance, as analyzed in Remark 1. Besides, a higher SE is obtained when the relay either moves towards the source or users. It is because the relay can harvest sufficient energy from the source when it is close to the source, or reduces PL of relay-users link when it is close to the users. The observation is essentially different from the conventional
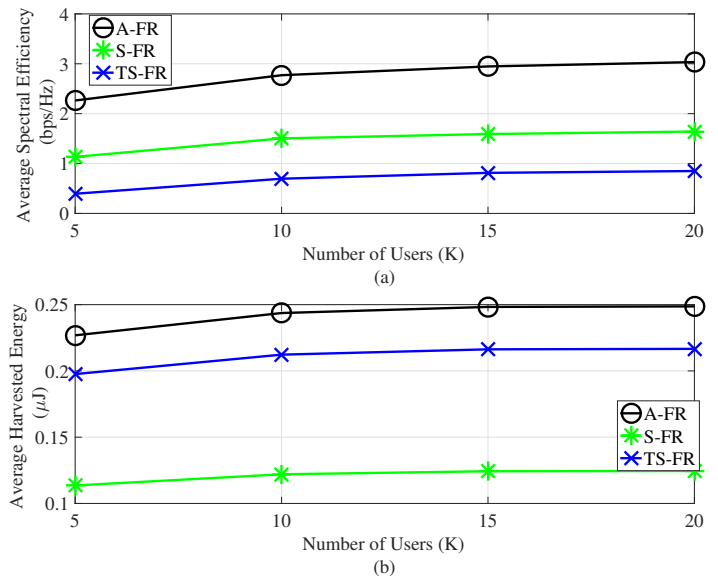

Fig. 6. Impact of the number of the users on SE performance and harvested energy, with $d_{S D}=d_{R D}=50 \mathrm{~m}$ and $p_{s}=30 \mathrm{dBm}$.

power-grid powered relaying system, where the maximum SE is normally achieved when the relay is positioned in the middle between the source and users.

Fig. 6 (a) demonstrates the end-to-end SE with different numbers of users. The A-FR system outperforms the two benchmarks with different number of users in terms of SE performance, as the proposed scheme has a better utilization of the limited system resources. Besides, benefiting from the multiuser diversity, all the three algorithms achieve higher $\mathrm{SE}$ with the increased number of users. The similar trend is observed from Fig. 6 (b), where more energy is harvested benefiting from the multiuser diversity. However, it can be seen that the harvested energy can not always increase with the number of users. It is because the harvested energy depends on the input power $p_{i n}$, which is a function of transmission power $p_{s, k, n}, p_{r, k, n}$ and propagation channels (as indicated by Eq. (9)). Although channel diversity is improved with more users and the system can find the channels with better conditions to carry signal, the summation of $p_{s, k, n}$ and $p_{r, k, n}$ over all subcarriers and users can not be always increased. As a result, the increase of overall harvested energy is trivial when the number of user is high enough.

Fig. 7 shows the SE performance under channel estimation error, where the SE is calculated based on the estimated channel. It can be seen that, the SE of all the three algorithms increases with a more accurate channel estimation, and the proposed A-FR algorithm always outperforms the benchmarks. Moreover, given an achievable value of channel estimation error [44], such as $10^{-4}$, the SE loss is trivial and the proposed algorithm maintains a favorable SE performance, compared to the perfect channel estimation scenario.

\section{B. Harvested Energy}

Fig. 8 demonstrates the average harvested energy by the three systems with different transmission power. The proposed A-FR system harvests the largest amount of energy, and achieves at least 50\% improvement than the S-FR system, when the transmission power budget at the source $p_{\max }$ ranges 


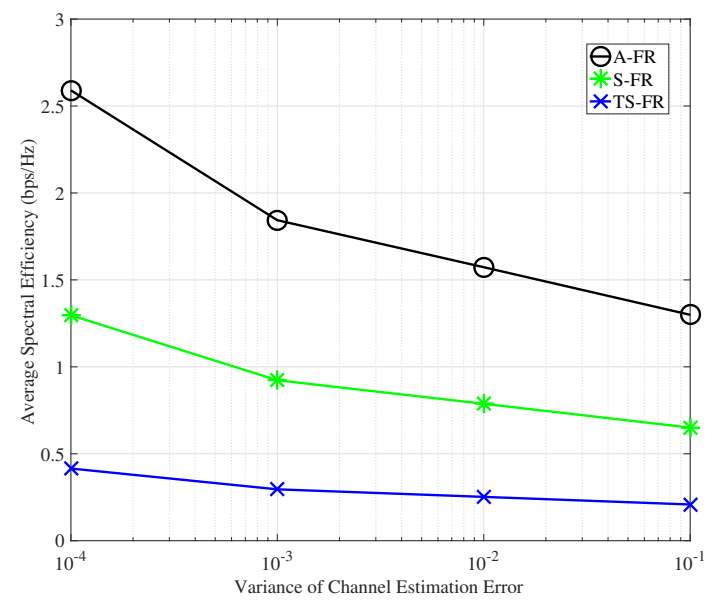

Fig. 7. Impact of channel estimation error $\sigma_{e}^{2}$ on SE performance with $d_{S D}=$ $d_{R D}=50 \mathrm{~m}$ and $p_{s}=30 \mathrm{dBm}$.

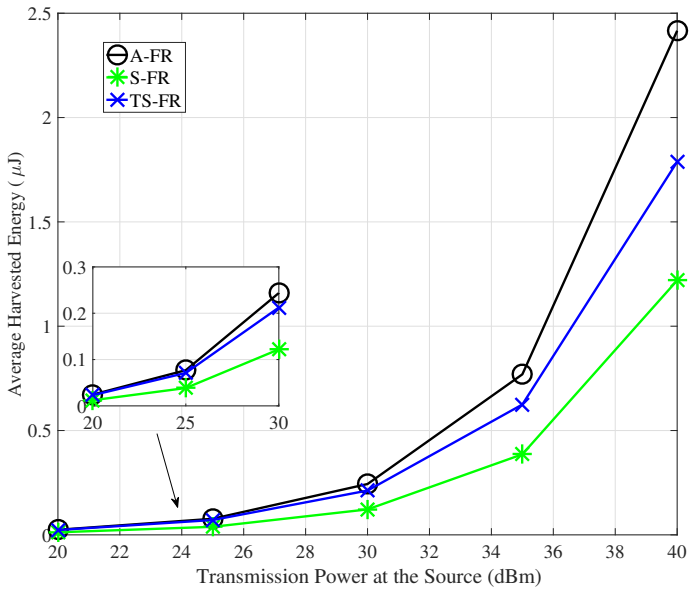

Fig. 8. Impact of the transmission power on the energy harvested with $d_{S R}=$ $d_{R D}=50 \mathrm{~m}$.

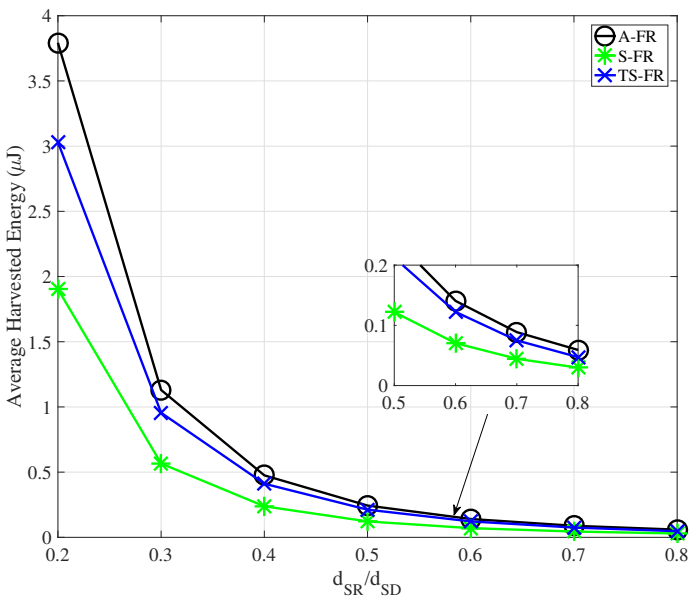

Fig. 9. Impact of the normalized source-relay distance on the energy harvested with $d_{S D}=100 \mathrm{~m}$ and $p_{s}=30 \mathrm{dBm}$.

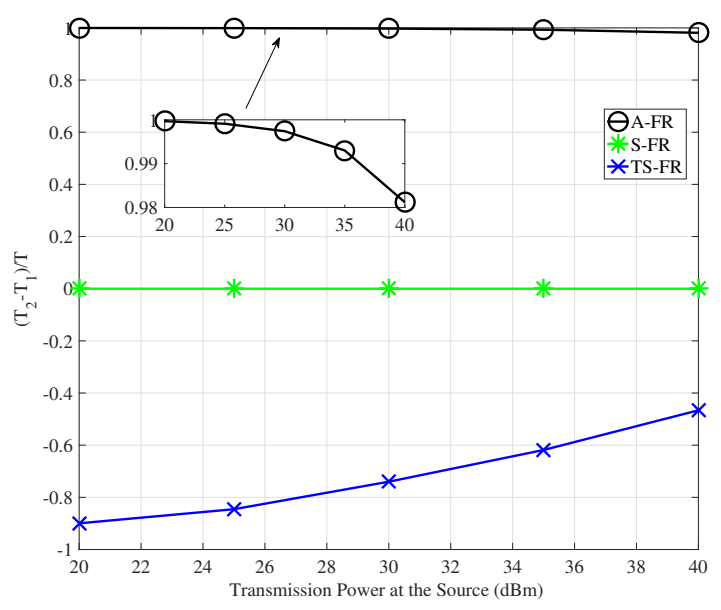

Fig. 10. Impact of the transmission power on the value of $\left(T_{2}-T_{1}\right) / T$ with $d_{S R}=d_{R D}=50 \mathrm{~m}$

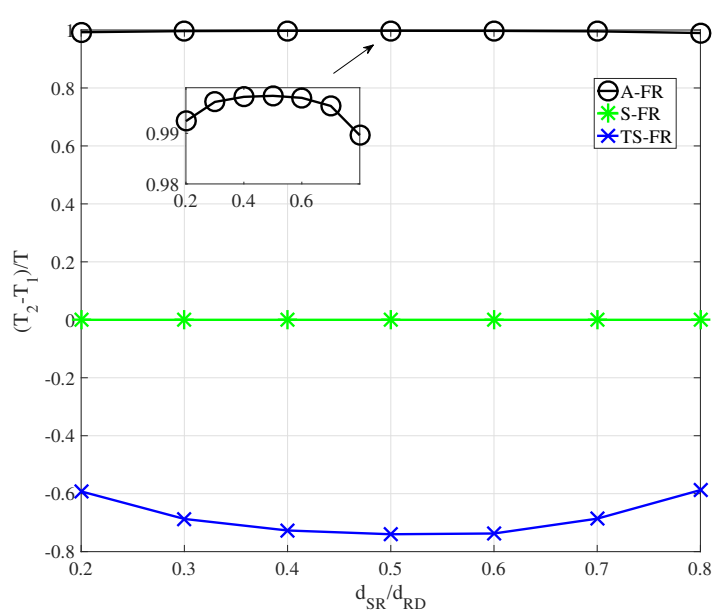

Fig. 11. Impact of the normalized source-relay distance on the value of $\left(T_{2}-T_{1}\right) / T$ with $d_{S D}=100 \mathrm{~m}$ and $p_{s}=30 \mathrm{dBm}$.

from $20-40 \mathrm{dBm}$. It is because the A-FR system allocates a longer duration for the second time slot $T_{2}$ to compensate the weak SINR at user end, allowing the relay to have more time to harvest energy concurrently. Besides, by the proposed the A-FR scheme, wireless energy originates from both the source and relay, which is naturally superior to the TS-FR system. As a comparison, the TS-FR system solely harvests energy from the source, whereas it allocates more time $\left(T_{1}\right.$ is used for EH operation by the TS-FR) for EH operation at the relay. Therefore, it can harvest more energy over the symmetric SFR scheme but is still inferior to the proposed A-FR.

Fig. 9, on the other hand, shows more energy can be harvested by the proposed A-FR system with different relay's locations. Besides, the harvested wireless energy decreases given a longer source-relay distance due to the significant signal attenuation. 

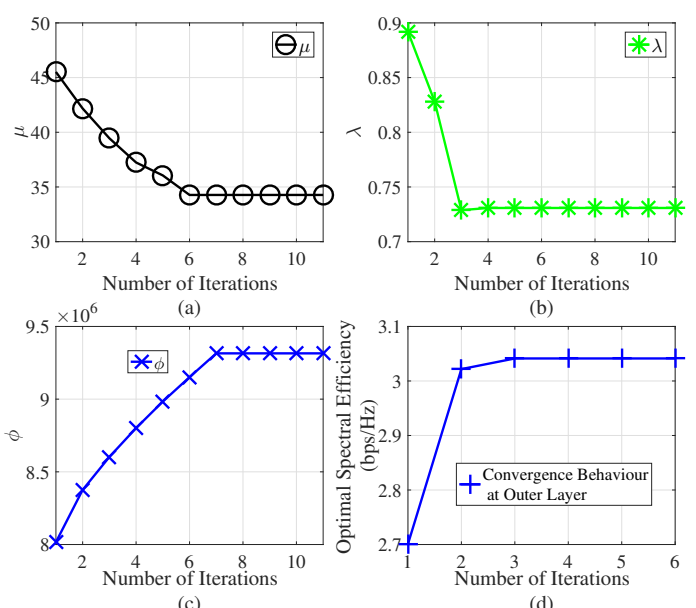

(d)

Fig. 12. Iteration behavior on finding $\lambda^{*}, \mu^{*}$, and $\phi^{*}$, where $d_{S R}=d_{R D}=$ $50 \mathrm{~m}$ and $p_{s}=30 \mathrm{dBm}$.

\section{Time Duration}

Fig. 10 shows the value of $\left(T_{2}-T_{1}\right) / T$ with different transmission power at the source. In the A-FR system, the length of the first time slot $T_{1}$ is slightly increased with a higher transmission power at the source. This is because in this case more energy is obtained by the relay and the effective SINR of the second hop is also improved. The system needs to reduce the length of the second time slot $T_{2}$ and replenish it to the first time slot $T_{1}$. On the contrary, $T_{1}$ decreases with a higher transmission power at the source. In fact, time slot $T_{1}$ is only used for energy harvesting in the TS-FR system. Hence, wireless energy may be collected quickly given a higher transmission power, and the system can spare more time to $T_{2}$ for information transmission. At last, the S-FR system allocates uniform time slots and is unaware of relay's positions, and thus its SE of the first link is wasted partially.

Fig. 11 demonstrates the value of $\left(T_{2}-T_{1}\right) / T$ with different relay's positions. In the A-FR system, when the relay moves to the middle point, the time slot $T_{1}$ is slightly decreased due to the reduced $\mathrm{EH}$ power at the relay, and the system has to arrange a larger value for $T_{2}$ to remedy the low SINR of the relay-user link. While if the distance of the relay-users link is much shorter than that of the first link, the time slot $T_{1}$ begins to increase because the SINR of the source-relay is significantly degraded with a longer source-relay distance. Therefore, system needs to increase the value of $T_{1}$ to rebalance the SE of the two links. As comparisons, the S-FR system has uniform time slot duration, which is independent of the relay's position. While the TS-FR system shows an opposite trend compared to the A-FR algorithm, caused by the different information transmission and $\mathrm{EH}$ mechanism.

\section{Convergence Behavior and EE Performance}

Figs. 12 (a)-(c) shows an example of the iteration behavior of the Lagrange multipliers $\mu, \lambda$, and $\phi$, in the inner layer. It can be observed that the proposed A-FR algorithm updates the multipliers iteratively, and the optimal values of the multipliers

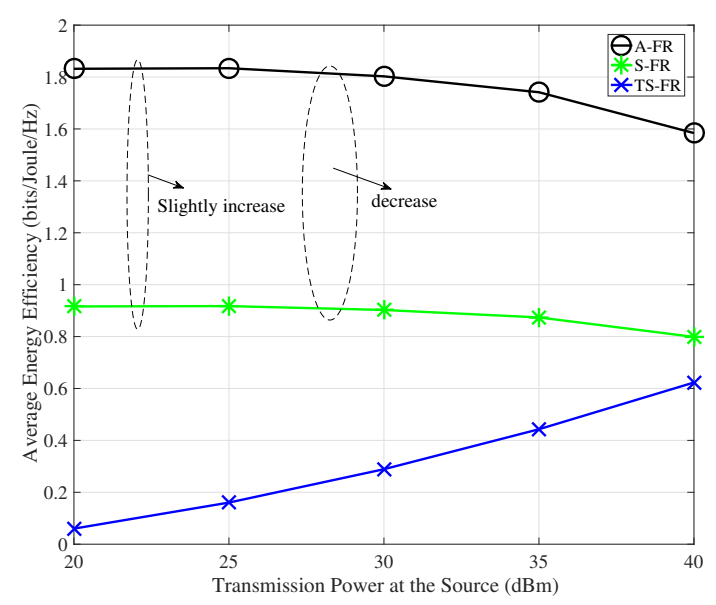

Fig. 13. Impact of the transmission power on EE performance, where $d_{S R}=$ $d_{R D}=50 \mathrm{~m}$

are converged within a few iterations. On the other hand, Fig. 12 (d) shows the convergence behavior in the outer layer for updating $\boldsymbol{p}_{s}^{(j)}$ and $\boldsymbol{p}_{r}^{(j)}$. Since successive convex approximation is applied to transform the original constraint (C4) into a relaxed constraint $(\tilde{C} 4)$, we need to update the value of $\boldsymbol{p}_{\boldsymbol{s}}^{(j)}$ and $\boldsymbol{p}_{\boldsymbol{r}}^{(j)}$ in the outer layer to make the approximation tight. As can be seen, at most 4-6 iteration endorses a convergence in the outer layer. In summary, the proposed algorithm iteratively runs in a two-lay structure and fast convergence can be confirmed.

Fig. 13 demonstrates the resulting energy efficiency (EE) performance with optimized end-to-end SE and different transmission power configurations at the source. To be more specific, the optimized SE is obtained by solving P3, subject to constraints $(\tilde{C} 1)-(\tilde{C 8})$, and then EE is calculated as the ratio of SE to the total power consumption. Note that an EE maximization problem that involves both SE and power consumption can be handled by quasi-concave optimization. That is, one can transform the fractional structure into a more tractable difference structure, which can be solved by standard convex optimization approaches [47] [48]. As seen, the proposed A-FR obtains the highest EE while the TSFR is the least efficient solution among the algorithms. In particular, the EE of the proposed algorithm slightly increases with transmission power ranging from $20 \mathrm{dBm}$ to $25 \mathrm{dBm}$, and begins to decrease if transmission power keeps increasing. It is because $\mathrm{EE}$ is generally quasi-concave with respect to the power consumption [45] [46], indicating that utilizing high transmission power may not lead to the highest EE. For SE maximization problem, it aims to achieve a high SE regardless of the power consumption, and hence all the transmission power is fully utilized by the source. As a result, its EE is not always increased because the power consumption in the denominator is also boosted, and the same trend is observed by the S-FR algorithm. At last, for the TS-FR algorithm, although its SE keeps increasing when transmission power is lower than $40 \mathrm{dBm}$, it can be expected that its EE may decrease with a higher transmission power. 


$$
T_{1}=\frac{T \sum_{k=1}^{K} \sum_{n=1}^{N} \beta_{k, n} \log _{2}\left(1+\frac{p_{r, k, n} \gamma_{r d, k, n}}{\beta_{k, n}}\right)}{\sum_{k=1}^{K} \sum_{n=1}^{N} \beta_{k, n} \log _{2}\left(1+\frac{p_{s, k, n} \gamma_{s r, k, n}}{\beta_{k, n}}\right)+\sum_{k=1}^{K} \sum_{n=1}^{N} \beta_{k, n} \log _{2}\left(1+\frac{p_{r, k, n} \gamma_{r d, k, n}}{\beta_{k, n}}\right)},
$$

$$
T_{2}=\frac{T \sum_{k=1}^{K} \sum_{n=1}^{N} \beta_{k, n} \log _{2}\left(1+\frac{p_{s, k, n} \gamma_{s r, k, n}}{\beta_{k, n}}\right)}{\sum_{k=1}^{K} \sum_{n=1}^{N} \beta_{k, n} \log _{2}\left(1+\frac{p_{s, k, n} \gamma_{s r, k, n}}{\beta_{k, n}}\right)+\sum_{k=1}^{K} \sum_{n=1}^{N} \beta_{k, n} \log _{2}\left(1+\frac{p_{r, k, n} \gamma_{r d, k, n}}{\beta_{k, n}}\right)} .
$$

$$
\max _{T_{1}, T_{2}, \boldsymbol{p}_{\boldsymbol{s}}, \boldsymbol{p}_{\boldsymbol{r}}, \boldsymbol{\beta}} \frac{2\left(\sum_{k=1}^{K} \sum_{n=1}^{N} \beta_{k, n} \log _{2}\left(1+\frac{p_{s, k, n} \gamma_{s r, k, n}}{\beta_{k, n}}\right)\right)\left(\sum_{k=1}^{K} \sum_{n=1}^{N} \beta_{k, n} \log _{2}\left(1+\frac{p_{r, k, n} \gamma_{r d, k, n}}{\beta_{k, n}}\right)\right)}{\sum_{k=1}^{K} \sum_{n=1}^{N} \beta_{k, n} \log _{2}\left(1+\frac{p_{s, k, n} \gamma_{s r, k, n}}{\beta_{k, n}}\right)+\sum_{k=1}^{K} \sum_{n=1}^{N} \beta_{k, n} \log _{2}\left(1+\frac{p_{r, k, n} \gamma_{r d, k, n}}{\beta_{k, n}}\right)} .
$$

\section{CONCLUSION}

To address the unbalanced SE performances of the sourcerelay and the relay-users hops in WPT-aided relaying systems, a novel dynamic structure has been proposed to allow nonuniform time slot durations for the two hops, achieving a better utilization of the degrees of freedom than the symmetric systems (S-FR) [27] and the time-switching based systems (TS-FR) [23]. A practical non-linear EH circuit has been considered, and an optimal algorithm has been developed to optimize the system end-to-end SE, by jointly designing time slot durations, transmission power at the source and the relay, and subcarriers for multiple users. Closed-forms of the optimal results have been derived. Simulation has showed that the proposed A-FR system can achieve higher SE than the S-FR [27] and TS-FR [23] systems. Benefiting from dynamic time slot allocation and utilizing the self-interference for $\mathrm{EH}$ operation, the energy harvested by the proposed AFR system is much higher than that of the T-FR and SFR systems, respectively, showing its high energy efficiency and applicability to WPT-assisted relaying systems. At last, an intensive properties discussion has been presented. It has been shown that the second time slot duration $T_{2}$ is normally larger than the first time slot duration $T_{1}$, since the available transmission power at the relay is significantly lower than that at the source caused by high PL and low EH efficiency. It also has been shown that, by harvesting energy from selfinterference signal rather than suppressing it, more power is assigned to the subcarriers having stronger self-interference channel gain. In contrast, conventional grid-powered FD relay systems [41], [42] assign more power to the subcarriers having weaker self-interference channel gain.

\section{APPENDIX A}

\section{PROOF OF PROPOSITION 1}

Using (C5) and (C8), $T_{1}$ and $T_{2}$ can be calculated by Eqs. (35) and (36), respectively. Substituting (35) and (36) into (9), we get an equivalent objective function as express by Eq. (37). Then the objective function can be transformed into

$$
\begin{aligned}
\min _{T_{1}, T_{2}, \boldsymbol{p}_{\boldsymbol{s}}, \boldsymbol{p}_{\boldsymbol{r}}, \boldsymbol{\beta}} & \frac{1}{2}\left(\frac{1}{\sum_{k=1}^{K} \sum_{n=1}^{N} \beta_{k, n} \log _{2}\left(1+\frac{p_{s, k, n} \gamma_{s r, k, n}}{\beta_{k, n}}\right)}+\right. \\
& \left.\frac{1}{\sum_{k=1}^{K} \sum_{n=1}^{N} \beta_{k, n} \log _{2}\left(1+\frac{p_{r, k, n} \gamma_{r d, k, n}}{\beta_{k, n}}\right)}\right) .
\end{aligned}
$$

Consider two inter functions $u\left(T_{1}, T_{2}, \boldsymbol{p}_{\boldsymbol{s}}, \boldsymbol{p}_{\boldsymbol{r}}, \boldsymbol{\beta}\right)=$ $\sum_{k=1}^{K} \sum_{n=1}^{N} \beta_{k, n} \log _{2}\left(1+\frac{p_{s, k, n} \gamma_{s, k, n}}{\beta_{k, n}}\right) \quad$ and $v\left(T_{1}, T_{2}, \boldsymbol{p}_{\boldsymbol{s}}, \boldsymbol{p}_{\boldsymbol{r}}, \boldsymbol{\beta}\right)=\sum_{k=1}^{K} \sum_{n=1}^{N} \beta_{k, n} \log _{2}\left(1+\frac{p_{r, k, n} \gamma_{r d, k, n}}{\beta_{k, n}}\right)$. The Hessian matrix of $u\left(T_{1}, T_{2}, \boldsymbol{p}_{\boldsymbol{s}}, \boldsymbol{p}_{\boldsymbol{r}}, \boldsymbol{\beta}\right)$ is given as

$$
\begin{aligned}
& \boldsymbol{H}\left(u\left(T_{1}, T_{2}, \boldsymbol{p}_{\boldsymbol{s}}, \boldsymbol{p}_{\boldsymbol{r}}, \boldsymbol{\beta}\right)\right)= \\
& \frac{p_{s, k, n} \gamma_{s r, k, n}^{2}}{\left(\beta_{k, n}+p_{s, k, n} \gamma_{s r, k, n}\right)^{2}}\left(\begin{array}{cc}
-\frac{p_{s, k, n}}{\beta_{k, n}} & 1 \\
1 & -\frac{\beta_{k, n}}{p_{s, k, n}}
\end{array}\right) .
\end{aligned}
$$

It can be seen that (39) is a negative semi-definite matrix, and hence $u\left(T_{1}, T_{2}, \boldsymbol{p}_{\boldsymbol{s}}, \boldsymbol{p}_{\boldsymbol{r}}, \boldsymbol{\beta}\right)$ is a concave function. Also, the Hessian matrix of function $v\left(T_{1}, T_{2}, \boldsymbol{p}_{\boldsymbol{s}}, \boldsymbol{p}_{\boldsymbol{r}}, \boldsymbol{\beta}\right)$ is given as

$$
\begin{aligned}
& \boldsymbol{H}\left(v\left(T_{1}, T_{2}, \boldsymbol{p}_{\boldsymbol{s}}, \boldsymbol{p}_{\boldsymbol{r}}, \boldsymbol{\beta}\right)\right)= \\
& \frac{p_{r, k, n} \gamma_{r d, k, n}^{2}}{\left(\beta_{k, n}+p_{r, k, n} \gamma_{r d, k, n}\right)^{2}}\left(\begin{array}{cc}
-\frac{p_{r, k, n}}{\beta_{k, n}} & 1 \\
1 & -\frac{\beta_{k, n}}{p_{r, k, n}}
\end{array}\right),
\end{aligned}
$$

which is a negative semi-definite matrix, and hence $v\left(T_{1}, T_{2}, \boldsymbol{p}_{\boldsymbol{s}}, \boldsymbol{p}_{\boldsymbol{r}}, \boldsymbol{\beta}\right)$ is a concave function. Since $u\left(T_{1}, T_{2}, \boldsymbol{p}_{\boldsymbol{s}}, \boldsymbol{p}_{\boldsymbol{r}}, \boldsymbol{\beta}\right)$ and $v\left(T_{1}, T_{2}, \boldsymbol{p}_{\boldsymbol{s}}, \boldsymbol{p}_{\boldsymbol{r}}, \boldsymbol{\beta}\right)$ are both concave and positive, we conclude that the equivalent function $\frac{1}{2}\left(\frac{1}{u}+\frac{1}{v}\right)$ in (39) is a convex function. It further indicates that the objective function in P3 is jointly concave with respect to the variables.

\section{REFERENCES}

[1] D. Niyato, D. I. Kim, M. Maso, and Z. Han, "Wireless Powered Communication Networks: Research Directions and Technological Approaches," IEEE Wireless Commun., vol. 24, no. 6, pp. 88-97, Dec.2017.

[2] B. Clerckx, R. Zhang, R. Schober, D. W. K. Ng, D. I. Kim, and H. V. Poor, "Fundamentals of Wireless Information and Power Transfer: From RF Energy Harvester Models to Signal and System Designs," IEEE J. Select. Areas in Commun., vol. 37, no. 1, pp. 4-33, Jan. 2019. 
[3] Z. Wei, S. Sun, X. Zhu, Y. Huang, L. Dong, and D. I. Kim, "Wireless information and power transfer: spectral efficiency optimization for asymmetric full-duplex relay systems (invited)," IEEE Proc. VTC Spr., Sydney, Australia, Jun. 2017.

[4] S. Li, W. Li, J. Deng, and C. C. Mi, "A double-sided LCC compensation network and its tuning method for wireless power transfer," IEEE Trans. Veh. Technol., vol. 64, no. 6, pp. 2261-2273, Jun. 2015.

[5] I. Krikidis, S. Timotheou, S. Nikolaou, G. Zheng, D. W. K. Ng, and R. Schober, "Simultaneous wireless information and power transfer in modern communication systems," IEEE Commun Mag., vol. 52, no. 11, pp. 104-110, Nov. 2014.

[6] J. Wang, C. Jiang, Z. Han, Y. Ren, and L. Hanzo, "Network association strategies for an energy harvesting aided super-WiFi network relying on measured solar activity," IEEE J. Sel. Areas Commun., vol. 34, no. 12, pp. 3785-3797, Dec. 2016.

[7] Q. Li and J. Qin, "Secure relay beamforming for simultaneous wireless information and power transfer in non-regenerative relay networks," IEEE Trans. Veh. Technol., vol. 63, no. 5, pp. 2462-2467, Jun. 2014

[8] D. Mishra, S. De, and D. Krishna, "Dilemma at RF energy harvesting relay: downlink energy relaying or uplink information transfer," IEEE Trans. Wireless Commun., vol. 16, no. 8, pp. 4939-4955, Aug. 2017.

[9] D. Mishra and S. De, "Integrated information relay and energy supply assisted RF harvesting communication," IEEE Trans. Commun., vol. 65, no. 3, pp. 1274-1288, Mar. 2017.

[10] A. A. Nasir, X. Zhou, S. Durrani, and R. Kennedy, "Relaying protocols for wireless energy harvesting and information processing," IEEE Trans. Wireless Commun., vol. 12, no. 7, pp. 3633-3636, Jul. 2013.

[11] Z. Ding, I. Krikidis, and H. V. Poor, "Wireless information and power transfer in cooperative networks with spatially random relaying," IEEE Trans Wireless Commun., vol. 13, no. 8, pp. 4440-4453, Aug. 2014.

[12] S. Atapattu and C. Tellambura, "Time-switching energy harvesting in relay networks," in Proc. IEEE ICC 15., London, UK, Jun. 2015.

[13] G. Amarasuriya, E. G. Larsson, and H. V. Poor, "Wireless Information and Power Transfer in Multiway Massive MIMO Relay Networks," IEEE Trans Wireless Commun., vol. 15, no. 6, pp. 3837-3855, Jun. 2016.

[14] S. Atapattu and J. Evans, "Optimal power-splitting ratio for wireless energy harvesting in relay networks," in Proc. IEEE VTC-Fall 15., Boston, USA, Sep. 2015.

[15] S. Atapattu and J. Evans, "Optimal energy harvesting protocols for wireless relay networks," IEEE Trans. on Wireless Commun., vol. 15, no. 8, pp. 5789-5803, Aug. 2016.

[16] Z. Wei, X. Zhu, S. Sun, Y. Huang, L. Dong, and Y. Jiang, "Full-duplex vs. half-duplex amplify-and-forward relaying: which is more energy efficient in $60 \mathrm{GHz}$ dual-hop indoor wireless systems?" IEEE J. Sel. Areas Commun., vol. 33, no. 12, pp. 2936-2947, Dec. 2015.

[17] M. M. Razlighi and N. Zlatanov, "Buffer-aided relaying for the two-hop full-duplex relay channel with self- interference," IEEE Transactions on Wireless Commun., vol. 17, no. 1, pp. 477-491, Jan. 2018.

[18] N. Zlatanov, R. Schober, and P. Popovski, "Buffer-aided relaying with adaptive link selection," IEEE J. Select. Areas Commun., vol. 31, no. 8, pp. 1530-1542, Aug. 2013.

[19] D. W. K. Ng, E. S. Lo and R. Schober, "Dynamic resource allocation in MIMO-OFDMA systems with full-duplex and hybrid relaying," IEEE Trans. Commun., vol. 60, no. 5, pp. 1291-1304, May 2012.

[20] R. Saini, D. Mishra, and S. De, "Utility regions for DF relay in OFDMAbased secure communication with untrusted users," IEEE Commun. Lett., vol. 21, no. 11, pp. 2512-2515, Nov. 2017.

[21] H. Ju and R. Zhang, "Optimal resource allocation in full-duplex wirelesspowered communication network," IEEE Trans. Commun., vol. 62, no. 10, pp. 3528-3540, Oct. 2014.

[22] T. Riihonen, L. Zhao, M. Vehkaper, and X. Wang, "On the feasibility of full-duplex relaying powered by wireless energy transferm" in Proc. IEEE SPAWC'16, Edinburgh, UK, Jul. 2016.

[23] C. Zhong, H. A. Suraweera, G. Zheng, I. Krikidis, and Z. Zhang, "Wireless information and power transfer with full duplex relaying," IEEE Trans. Commun., vol. 62, no. 10, pp. 3447-3461, Oct. 2014.

[24] M. Mohammadi, B. K. Chalise, H. A. Suraweera, C. Zhong, G. Zheng, and I. Krikidis, "Throughput analysis and optimization of wirelesspowered multiple antenna full-duplex relay systems ," IEEE Trans. Commun., vol. 64, no. 4, pp. 1769-1785, Apr. 2016.

[25] Z. Wen, X. Liu, N. C. Beaulieu, R. Wang, and S. Wang, "Joint source and relay beamforming design for full-duplex MIMO AF relay SWIPT systems," IEEE Commun. Lett., vol. 20, no. 2, pp. 320-323, Feb. 2016.

[26] D. Hwang, K. C. Hwang, D. I. Kim, and T. J. Lee, "Self-energy recycling for RF powered multi-antenna relay channels," IEEE Trans Wireless Commun., vol. 16, no. 2, pp. 812-824, Feb. 2017.
[27] Y. Zeng and R. Zhang, "Full-duplex wireless-powered relay with selfenergy recycling," IEEE Wireless Commun. Lett., vol. 4, no. 2, pp. 201203, Apr. 2015.

[28] H. Li, N. Jaggi, and B. Sikdar, "Relay scheduling for cooperative communications in sensor networks with energy harvesting," IEEE Trans. Wireless Commun., vol. 10. no. 9, pp. 2918-2928, Sept. 2011.

[29] L. Guo, X. Ding, H. Wang, Q. Li, S. Chen, and X. Zhang, "Cooperative relay service in a wireless LAN," IEEE J. Sel. Areas Commun.,vol. 25, no. 2, pp. 355-368, Feb. 2007.

[30] K. W. Choi, D. Setiawan, J. Park, S. I. Hwang, D. Kang, M. Chung, and D. I. Kim, "Towards realization of long-range wireless-powered sensor networks," accepted by IEEE Wireless Commun., Mar. 2019.

[31] Y. Zeng and R. Zhang, "Optimized training design for wireless energy transfer," IEEE Trans. Commun., vol. 63, no. 2, pp. 536-550, Feb. 2015.

[32] B. Clerckx and E. Bayguzina, "Waveform design for wireless power transfer," IEEE Signal Process. Lett., vol. 64, no. 23, pp. 6313-6328, Dec. 2016.

[33] E. Boshkovsha, D. W. K. Ng, N. Zlatanov, and R. Schober, "Practical non-linear energy harvesting model and resource allocation for SWIPT systems," IEEE Commun Lett., vol. 19, pp. 2082-2085, Dec. 2015.

[34] M. Maso, C. Liu, C. Lee, T. Quek, and L. Cardoso, "Energy-recycling full-duplex radios for next generation networks," IEEE J. Sel. Areas Commun., vol. 33, no. 12, pp. 2948-2962, Dec. 2015.

[35] N. Zhou, X. Zhu, J. Gao, and Y. Huang, "Optimal asymmetric resource allocation with limited feedback for OFDM based relay systems," IEEE Trans. Wireless Commun., vol. 9, no. 2, pp. 552-557, Feb. 2010.

[36] Y. Zeng, H. Chen, and R. Zhang, "Bidirectional wireless information and power transfer with a helping relay," IEEE Wireless Commun. Lett., vol. 20, no. 5, pp. 862-865, May 2016 .

[37] Wei. Y and R. Lui, "Dual methods for nonconvex spectrum optimization of multicarrier systems,"IEEE Trans. Commun., vol. 54, no. 7, pp. 13101322, Jul. 2006

[38] D. W. K. Ng, and R. Schober, "Energy-efficient resource allocation in OFDMA systems with large number of BS antennas," IEEE Trans. Wireless Commun., vol. 11, no. 9, pp. 3292-3304, Sep. 2012.

[39] D. W. K. Ng, and R. Schober, "Cross-layer scheduling for OFDMA amplify and forward relay networks," IEEE Trans. Veh. Tech., vol. 59, no. 3, pp. 1443-1458, Mar. 2010.

[40] D. W. K. Ng, E. Lo, and R. Schober, "Wireless information and power transfer: energy efficiency optimization in OFDMA systems," IEEE Trans. Wireless Commun., vol. 12, no. 12, pp. 6352-6370, Dec. 2013.

[41] Z. Wei, X. Zhu, S. Sun, and Y. Huang, "Energy-efficiency-oriented crosslayer resource allocation for multiuser full-duplex decode-and-forward indoor relay systems at $60 \mathrm{GHz}$," IEEE J. Sel. Areas Commun., vol. 34, no. 12, pp. 3366-3379, Dec. 2016.

[42] Z. Wei, X. Zhu, S. Sun, A. Al-Tahmeesschi, and Y. Jiang "Energyefficiency of millimeter-wave full-duplex relaying systems: challenges and solutions," IEEE Access, vol. 4, pp. 4848-4860, Jul. 2016.

[43] D. Tse and P. Viswanath, "Fundamentals of wireless communication," Cambridge University Press, First Edition, 2004.

[44] F. Gao, R. Zhang, and Y. Liang, "Channel estimation for OFDM modulated two-way relay networks," IEEE Trans. Signal Process., vol. 57, no. 11, pp. 4443-4455, Nov. 2009.

[45] G. Miao, N. Himayat, and G. Y. Li, "Energy-efficient link adaptation in frequency-selective channels," IEEE Trans. Commun., vol. 58, no. 2, pp. 545-554, Feb. 2010.

[46] X. Xiao, X. Tao, and J. Lu, "Energy-efficient resource allocation in LTE based MIMO-OFDMA systems with user rate constraints," IEEE Trans. Veh. Technol., vol. 64, no. 1, pp. 185-197, Jan. 2015.

[47] Z. Wei, X. Zhu, S. Sun, J. Wang, and L. Hanzo, "Energy-efficient fullduplex cooperative non-orthogonal multiple access," IEEE Trans. Veh. Technol., vol. 67, no. 10, pp. 10123-10128, Sep. 2018.

[48] S. Boyd and L. Vandenberghe, Convex Optimization. Cambridge, U.K.: Cambridge Univ. Press, 2004. 


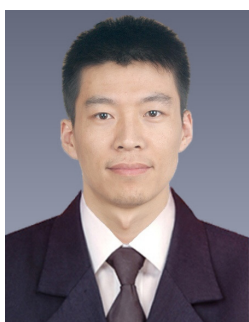

Zhongxiang Wei (S'15-M'17) is research associate of Electrical and Electronics Engineering at University College London, U.K. He received the $\mathrm{Ph} . \mathrm{D}$. degree from the University of Liverpool, Liverpool, U.K., in 2017. From March 2016 to March 2017, he was with the Institution for Infocomm Research, Agency for Science, Technology, and Research (A*STAR), Singapore, as a Research Assistant. From March 2017 to October 2017, he was a Research Assistant with the Wireless Networks and Communications Group, Harbin Institute of Technology (HIT), Shenzhen, China. His research interests include constructive interference design, green communications, full-duplex, millimeterwave communications, and algorithm design. He was the recipient of the Graduate China National Scholarship Award in 2012, and the A*STAR Research Attachment Programme (ARAP) Studentship in 2016. He has acted as a TPC member or session chair for various international conferences, such as ICC 2019 and 2020. He was the recipient of exemplary reviewer of IEEE Transaction on Wireless Communications in 2016.

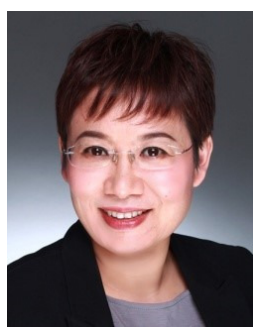

Sumei Sun (F'16) is currently Principal Scientist, Head of the Communications and Networks Cluster at the Institute for Infocomm Research (I2R), Lead Principal Investigator of the Industrial Internet of Things Research Program at Agency for Science, Technology, and Research (A*STAR), and an adjunct professor at the National University of Singapore. Her current research interests are in cognitive communications and networks, next-generation wireless communications, and industrial internet of things. She is a Distinguished Speaker of the IEEE Vehicular Technology Society 2018-2021, Vice Director of IEEE Communications Society Asia Pacific Board, and Chapter Coordinator of Asia Pacific Region in the IEEE Vehicular Technologies Society. She is also serving as a member of the IEEE Communications Society Globecom/ICC Management and Strategy (GIMS) Standing Committee, Inaugural Editor-in-Chief of IEEE Open Journal of Vehicular Technology, and member of the IEEE Transactions on Wireless Communications Steering Committee.

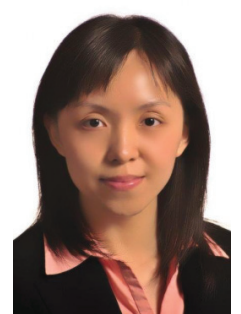

Xu Zhu (S'02-M'03-SM'12) received the B.Eng. degree (Hons.) in electronics and information engineering from the Huazhong University of Science and Technology, Wuhan, China, in 1999, and the $\mathrm{Ph} . \mathrm{D}$. degree in electrical and electronic engineering from The Hong Kong University of Science and Technology, Hong Kong, in 2003. She joined the Department of Electrical Engineering and Electronics, University of Liverpool, Liverpool, U.K., in 2003, as an Academic Member, where she is currently a Reader. She has authored or coauthored more than 180 peer-reviewed publications on communications and signal processing. Her research interests include MIMO, channel estimation and equalization, resource allocation, cooperative communications, and green communications. She has acted as a Chair for various international conferences, such as the Vice-Chair of the 2006 and 2008 ICARN International Workshops, the Program Chair of ICSAI 2012, the Symposium Co-Chair of the IEEE ICC 2016 and 2019, and the Publicity Chair of the IEEE IUCC 2016. She has served as an Editor for the IEEE TRANSACTIONS ON WIRELESS COMMUNICATIONS and a Guest Editor for several international journals such as Electronics.

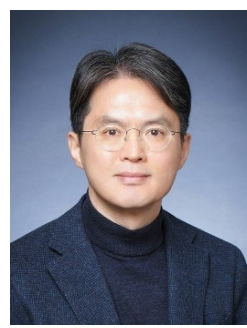

Dong In Kim (S'89-M'91-SM'02-F'19) received the $\mathrm{Ph} . \mathrm{D}$. degree in electrical engineering from the University of Southern California, Los Angeles, CA, USA, in 1990. He was a tenured Professor with the School of Engineering Science, Simon Fraser University, Burnaby, BC, Canada. Since 2007, he has been with Sungkyunkwan University, Suwon, South Korea, where he is currently a Professor with the College of Information and Communication Engineering. He has been elevated to the grade of Fellow of the IEEE for contributions to cross-layer design of wireless communications systems. He is also a Fellow of the Korean Academy of Science and Technology and a Member of the National Academy of Engineering of Korea. He is the Executive Chair for the IEEE ICC 2022 in Seoul. He was a first recipient of the NRF of Korea Engineering Research Center in Wireless Communications for RF Energy Harvesting (2014-2021). From 2001 to 2019, he served as an Editor of Spread Spectrum Transmission and Access and an Editor-at-Large of Wireless Communication I for the IEEE Transactions on Communications. From 2002 to 2011, he also served as an Editor and a Founding Area Editor of Cross-Layer Design and Optimization for the IEEE Transactions on Wireless Communications. From 2008 to 2011, he served as the Co-Editor-in-Chief for the IEEE/KICS Journal of Communications and Networks. He served as the Founding Editor-in-Chief for the IEEE Wireless Communications Letters, from 2012 to 2015.

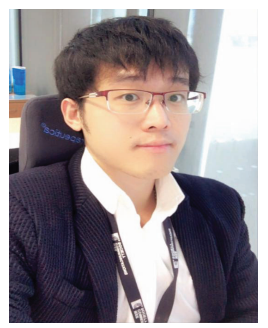

Derrick Wing Kwan Ng (S'06-M'12-SM'17) received the bachelor degree with first-class honors and the Master of Philosophy (M.Phil.) degree in electronic engineering from the Hong Kong University of Science and Technology (HKUST) in 2006 and 2008, respectively. He received his Ph.D. degree from the University of British Columbia (UBC) in 2012. He was a senior postdoctoral fellow at the Institute for Digital Communications, Friedrich-Alexander-University Erlangen-Nürnberg (FAU), Germany. He is now working as a Senior Lecturer and an ARC DECRA Research Fellow at the University of New South Wales, Sydney, Australia. His research interests include convex and non-convex optimization, physical layer security, wireless information and power transfer, and green (energy-efficient) wireless communications.

Dr. Ng received the Best Paper Awards at the IEEE TCGCC Best Journal Paper Award 2018, INISCOM 2018, IEEE International Conference on Communications (ICC) 2018, IEEE International Conference on Computing, Networking and Communications (ICNC) 2016, IEEE Wireless Communications and Networking Conference (WCNC) 2012, the IEEE Global Telecommunication Conference (Globecom) 2011, and the IEEE Third International Conference on Communications and Networking in China 2008. He has been serving as an editorial assistant to the Editor-in-Chief of the IEEE Transactions on Communications since Jan. 2012. In addition, he is listed as a Highly Cited Researcher by Clarivate Analytics in 2018 and 2019. 